\title{
RÉFLEXION ÉPISTÉMOLOGIQUE, SCIENCES SOCIALES ET GÉOGRAPHIE
}

\author{
Paul Claval ${ }^{1}$ \\ ${ }^{1}$ Université de Paris-Sorbonne
}

Recebido em 07/2013. Aceito para publicação em 10/2013.

Versão online publicada em 12/09/2014 (http://seer.ufrgs.br/paraonde)

\section{I- L'épistémologie : considérations générales}

\section{1- Un développement tardif}

On s'interroge depuis longtemps sur les fondements de la connaissance, mais l'épistémologie ne revêt qu'assez récemment ses formes modernes. En France, le tournant est pris avec Meyerson (1921). En Allemagne, le point de départ est plus ancien : Kant renouvelle l'analyse de la faculté de savoir.

La réflexion épistémologique tarde à s'affirmer dans les sciences de l'homme et de la société. Dans le courant du XIXe siècle, certains historiens allemands soulignent l'originalité de leur discipline, qui cherche à comprendre plus qu'à expliquer. C'est le point de départ du Methodenstreit, qui marque le vrai début de la réflexion sur les fondements des disciplines de l'homme et de la société.

\section{2- Une démarche fondée sur la philosophie ou sur} l'observation du travail scientifique

Le rôle dévolu à l'épistémologie varie selon les chercheurs. Certains y voient une super-discipline dont les fondements seraient philosophiques et qui indiquerait quelles démarches adopter, comment procéder au travail de terrain et quand et pourquoi construire une théorie. Pour d'autres, le propos est plus modeste : il est d'observer ce que font les spécialistes d'une science sociale, de le résumer, de le clarifier, de montrer les difficultés auxquelles se heurtent les démarches pratiquées et de faciliter ainsi la formation des étudiants.

Dans le premier cas, les chercheurs doivent se soumettre aux diktats de spécialistes d'un plus haut niveau ; ceux-ci sont soit des philosophes, soit des représentants de disciplines jugées plus avancées et que l'on prend comme modèles : des philosophes, des physiciens, des naturalistes, mais aussi des sociologues, des linguistes ou des spécialistes du discours.

Dans le second cas, l'épistémologie met en évidence ce qui se fait dans une discipline, souligne ce qui est nouveau dans ses démarches et dans ses façons de raisonner, fait apparaître les problèmes qu'elle rencontre, provoque des débats à leur sujet et facilite le progrès des connaissances.

Le danger de la première interprétation, c'est de conférer à une minorité un pouvoir intellectuel qui peut être excessif : celui de dénoncer les malfaçons dans les procédures de recherche sont entachées. La seconde interprétation risque de se montrer insuffisamment critique : elle suit au plus près ce qui se pratique, mais ne dit pas toujours ce que pourrait être la recherche et ce qu'elle devrait être.

\section{3- L'épistémologie comme tribunal des savoirs communs et des connaissances scientifiques}

Recoupant un peu cette seconde distinction, on peut en établir une troisième : celle qui considère que les sujets observés, mais aussi les chercheurs qui les étudient, souffrent d'une incapacité profonde à prendre conscience des motifs qui les guident et à mesurer les enjeux de ce qu'ils font. Le monde n'est-il pas peuplé d'individus irrationnels, hostiles au progrès et imbus de préjugés qui les empêchent de voir clairement les problèmes ? Les sciences de l'homme et de la société mettent en évidence ces faiblesses. Mais il faut mener plus loin cette analyse critique : les chercheurs peuvent également être frappés de myopie ou, plus grave, de cécité.

La recherche s'apparenterait ainsi à un système judiciaire dans lequel deux niveaux de tribunaux seraient nécessaires : le premier est celui qu'institue la science : celle-ci juge des actions des hommes, souligne leur irrationalité et propose une interprétation qui met en lumière les vrais mécanismes et le jeu des intérêts cachés ou inavoués; le second niveau est celui de l'épistémologie ; celle-ci soumet à son tour les chercheurs à un questionnement ; elle établit si leur travail est bien mené ; elle dénonce les erreurs qu'il comporte et les non-dits et préjugés qui entachent leurs résultats.

Une question se pose alors : deux instances suffisent-elles ? Dès lors que deux contrôles sont indispensables pour établir la vérité, un troisième, chargé de juger les juges de seconde instance, ne s'impose-t-il pas à son tour? 


\section{4- Une combinaison fréquente des deux approches}

La réflexion épistémologique est menée à la fois par des philosophes et par les praticiens des différentes disciplines scientifiques. Les premiers posent le problème du savoir en termes généraux : le réel existe-t-il ? L'homme peut-il vraiment le connaître ? A quelles conditions ? Les seconds ont des ambitions plus limitées : que peut-on savoir des corps, de la forme qu'ils revêtent, solide, liquide ou gazeuse, et de leurs diverses propriétés ? Quelles méthodes employer, l'observation ou l'expérimentation? Quels biais peuvent-ils invalider les résultats de l'observation? Comment mener les expériences, etc.?

Les progrès de la réflexion épistémologique résultent généralement de l'interaction de ces deux groupes. Les philosophes posent les problèmes de manière plus générale. Les résultats auxquels ils parviennent ont eu longtemps l'autorité de ce qui s'appuie sur la métaphysique. La situation change dans le courant du XVIIIe siècle, avec la révolution kantienne, qui coupe l'épistémologie de toute base métaphysique - mais la leçon de Kant n'est pas reçue par tous les philosophes, Hegel en particulier. Les philosophes s'appuient également sur l'autorité de la logique, avant que celle-ci ne devienne autonome, à la fin du XIXe siècle.

Les praticiens de la science ont sur les philosophes l'avantage d'affronter directement les problèmes que pose l'élaboration de la connaissance dans leur domaine particulier. C'est à eux que l'on doit les remises en cause, les réévaluations critiques, les révolutions scientifiques. C'est à la prise en compte de leurs difficultés et de leurs démarches que l'épistémologie philosophique doit de coller aux problèmes que pose vraiment l'élaboration de la connaissance. Les grands épistémologues ont d'ailleurs souvent une double formation : ils sont philosophes et physiciens, comme Gaston Bachelard $(1934$; 1938), philosophes et naturalistes, comme Canguilhem (1977), philosophes et sociologues...

\section{5-Certaines sciences servent de modèle aux autres}

Les modèles que se choisissent les savants varient avec le temps. Un exemple : comme tous les chercheurs en sciences humaines et sociales, les géographes ont longtemps considéré que les sciences exactes étaient plus évoluées ; à la primauté de l'astronomie, qui servait de guide aux géographes grecs ou à ceux de la Renaissance et des débuts de l'âge moderne se substitue la fascination pour la physique, dont les succès ne cessent de se confirmer à la suite de Newton. Le XVIIIe siècle y ajoute les sciences naturelles et le XIXe une des formes plus évoluée de celles-ci, la biologie. Le succès du darwinisme renforce l'influence des approches naturalistes et explique la naissance de la géographie humaine, qui, avec Ratzel (1882-1891), se définit comme une écologie de l'homme.

D'autres disciplines de la nature servent égale- ment de modèles : la géologie, qui inspire les géomorphologues, mais aussi les spécialistes de géographie régionale ; la botanique, qui en analysant ce qu'est une formation végétale éclaire les structures spatiales ; à partir d'un certain moment, c'est à la pédologie et surtout à l'écologie sous ses formes successives, sa variante énergétique et sa variante génique en particulier, que l'on a recours.

Les géographes regardent également du côté des sciences de la société et de l'homme. L'histoire, dont la position est dominante durant la première moitié du XIXe siècle, exerce une forte influence. Beaucoup de géographes, en France en particulier, ont d'ailleurs reçu une formation en ce domaine. La sociologie peut-elle servir de modèle ? La question est débattue avec passion à l'époque de Durkheim (1895) ; la conclusion est alors négative, mais une génération plus tard, l'attitude n'est plus la même grâce à la sociologie urbaine de Park et Burgess. Vis-à-vis de l'anthropologie et de l'ethnologie, les relations sont précoces et intenses, mais c'est plutôt la géographie qui sert d'inspiratrice comme on le voit avec Friedrich Ratzel et Franz Boas, ou avec l'anthropologie sociale anglaise de l'entre-deux-guerres.

Une nouvelle série de modèles s'impose après la Seconde Guerre mondiale. La science économique est alors la discipline reine. Elle s'intéresse depuis longtemps à l'économie spatiale : c'est de ce voisinage que naît la Nouvelle Géographie des années 1950 et 1960. Avec la vogue du structuralisme, les géographes se tournent vers de nouvelles formes d'anthropologie (celle de Lévi-Strauss, par exemple), d'histoire (la nouvelle histoire grecque, largement influencée par l'anthropologie et l'analyse de la longue durée) et de philosophie (la phénoménologie, grâce à l'influence de Heidegger, de Bachelard (1934 ; 1938), de Merleau-Ponty (1945), puis les écoles de la généalogie du savoir et de sa déconstruction, Foucault, Derrida, Deleuze, entre autres (sur cette école : Cusset, 2003).

\section{6- Progrès continu, révolutions scientifiques et tournants de la connaissance}

L'épistémologie classique cherchait à comprendre comment tel ou tel domaine pouvait être expliqué de manière rationnelle. A la vue statique d'une raison constituée depuis toujours se substitue progressivement l'idée que la rationalité scientifique se construit comme le montre Gaston Bachelard $(1934 ; 1938)$ - et qu'elle progresse par phases : des périodes d'additions successives sont interrompues par des remises en cause, des révolutions scientifiques, comme l'entrevoit Alexandre Koyré (1966) et le démontre.

L'idée que le progrès est cumulatif et continu domine jusqu'à la fin du XIXe siècle. Elle est alors remise en cause par l'évolution des mathématiques et l'élaboration des géométries non-euclidiennes. Elle l'est plus encore par l'évolution de la physique : théo- 
rie de la relativité, substitution à l'optique ondulatoire d'une optique corpusculaire. L'histoire des sciences fait un retour sur la révolution galiléenne.

Les sciences sociales se rallient un temps à la perspective kuhnienne, puis en adoptent une autre : elles mettent davantage l'accent sur les tournants disciplinaires ou transdisciplinaires que sur les révolutions scientifiques.

\section{II- L'épistémologie des sciences de l'homme et de la société : une grande diversité de démarches}

\section{1- Le développement tardif de l'épistémologie des scien- ces de l'homme et de la société}

Les sciences de l'homme et de la société sont des tard-venues. L'histoire et la géographie, font illusion, car elles se réclament d'un long passé, mais les orientations qu'elles prennent au XIXe siècle diffèrent de celles qu'elles suivaient jusqu'alors. La géographie faisait de l'orientation et de la représentation cartographique de la terre ses objets principaux - mais ceux-ci passent aux mains d'autres spécialistes lorsque, dans le courant du XVIIIe siècle, on parvient à mesurer de manière précise les longitudes. La discipline développe alors de nouvelles perspectives : la description et l'explication des formes du relief d'une part, l'analyse de la distribution des hommes et de leur empreinte à la surface de la terre de l'autre. L'histoire s'attachait à ce qui paraissait mémorable, à la vie des grands hommes, des conquérants, des capitaines illustres ; elle racontait les crises politiques. Elle devient étude des destins nationaux, avant de s'élargir, dans la seconde moitié du XXe siècle, à la globalité des peuples et de la terre.

Les sciences sociales naissent ou repensent leurs fondements dans le dernier tiers du XVIIIe siècle (l'économie ; la géographie naturelle), dans la première moitié du XIXe siècle (l'histoire, la sociologie, le folklore, la linguistique), dans la seconde moitié de ce siècle (l'ethnologie et l'anthropologie ; la géographie humaine) ou dans les premières décennies du XXe siècle (les sciences politiques). Il convient d'ajouter à cela des disciplines érudites, comme la philologie, la science des religions ou l'orientalisme, dont les contours s'affermissent au XIXe siècle, et qui jouent un grand rôle dans l'évolution générale des perspectives. La réflexion pédagogique se développe parallèlement à l'épistémologie et interfère parfois avec elle - comme le montre, en géographie, l'impact des idées de Rousseau et de Pestalozzi.

Il faut prendre également en compte l'évolution des disciplines plus étroites qui apparaissent dans le courant du XXe siècle : sciences de la communication, étude des formations discursives, étude du genre, praxéologie, etc. Le fossé qui séparait les sciences sociales des humanités se comble lorsque celles-ci prennent une forme plus systématique - en partie sous l'impact de la linguistique - à partir des années 1930.

\section{2- Une question fondamentale : qu'est-ce que la société?}

Une des questions fondamentales qui se posent aux sciences sociales est celle de la définition de leur objet central : la société. Plusieurs manières de la concevoir sont possibles. Dans le monde traditionnel, elle apparaît comme une entité qui enveloppe les individus, les forme, les guide et leur permet de vivre grâce à la culture dont elle les dote, grâce aux institutions qui structurent la vie collective et grâce à la solidarité qui s'impose à tous. La société fait en quelque sorte partie de la nature.

Le XVIIe siècle rompt avec cette conception. Sous l'influence de l'atomisme de Lucrèce, Thomas Hobbes (1651/1971) adopte une autre vue. L'individu préexiste à la société. Aucune solidarité : l'homme est un loup pour l'homme. C'est de l'insécurité qui en découle que résulte une décision fondamentale : celle de renoncer à la violence en signant un pacte, un covenant, un contrat social, qui délègue l'emploi de la force au Léviathan, c'est-à-dire à l'Etat. La société est donc une création volontaire ; elle est de nature politique. Elle s'organise en créant un espace homogène. Le Léviathan, qui domine celui-ci, est doté d'une souveraineté absolue sur la population et le territoire qu'il contrôle - la pensée de Hobbes est contemporaine du triomphe de l'Etat westphalien et de la géographie politique qui lui est liée. Au XVIIIe siècle, la théorie du droit naturel subit une inflexion : le peuple crée l'Etat au moment où il signe le contrat, mais reste détenteur de la légitimité, qu'il délègue aux gouvernants. L'Etat est donc l'expression politique de la nation.

Ces représentations permettent de fonder juridiquement les institutions politiques, mais la révolution française montre les limites de la démarche : l'impuissance des assemblées successives à construire un nouvel ordre républicain souligne que la société ne se fonde pas par décret: d'où l'émergence, au XIXe siècle d'une réflexion nouvelle sur la nature de la celle-ci.

Premier constat : société et culture préexistent à l'individu, le modèlent et le dépassent. Deuxième constat : la société crée des solidarités sans lesquelles la vie des individus serait impossible ; sans elles, les enfants ne seraient pas élevés, les personnes faibles aidées et les gens âgés secourus. La société naît d'un vouloir-vivre en commun ; celui-ci, renouvelé de génération en génération, rend vivant le lien social et crée une solidarité entre tous. Cette conception s'inspire du christianisme, puisqu'elle met la charité au premier plan, mais elle ne considère plus celle-ci comme une affaire individuelle et insiste sur sa dimension collective. C'est ainsi que se formule, entre 1800 et 1830 , l'idéal socialiste, inséparable de l'idée de solidarité.

Le sens que prend au XIXe siècle le terme de société s'oppose à celui que lui donnait le contrat social : il met au premier plan le collectif plutôt que l'individuel. 
La société est à la fois une réalité (puisque les hommes arrivent depuis toujours à vivre en commun) et une obligation (puisque pour être efficace, la solidarité doit être universelle et renouvelée). Dans la réalité, ces conditions ne sont qu'imparfaitement réalisées si bien que les sociétés observées sont parfois bancales. La science doit analyser les formes souvent boiteuses qu'elle inventorie et proposer des outils pour les réformer et renforcer le lien social.

\section{3- Une conséquence de la révolution industrielle : com- munauté et société}

La révolution industrielle transforme le monde. Dans le courant du XIXe siècle, l'idée que la société existait dans le monde traditionnel est partagée par beaucoup de chercheurs. Elle n'y était pas partout présente, c'est vrai ; là où la grande propriété dominait, deux univers sociaux s'opposaient, celui des grandes exploitants et celui des journaliers. Ces derniers étaient mal rémunérés, manquaient souvent d'emploi et se trouvaient réduits à la misère : un tel monde ignorait la solidarité.

La cohésion que crée le lien social s'observait en revanche dans les régions où la petite paysannerie était propriétaire de ses biens, soit individuellement, soit collectivement. Pour fonder une communauté, une société au sens le plus profond du terme, il est en effet indispensable d'assurer à la totalité ses membres les moyens de vivre décemment soit en leur distribuant des terres nouvelles, comme Jefferson l'imagine dans le cas des Etats-Unis, soit en maintenant la propriété collective des biens fonciers ou un contrôle collectif de leur utilisation.

Le mythe de la communauté agraire primitive s'installe ainsi. Ferdinand Tönnies (1887) va plus loin : face aux 'communautés' solidaires du monde traditionnel se dresse ce que il qualifie de 'société', et que façonnent la révolution industrielle et la modernité : un agglomérat d'individus qui ne sont plus liés que par la division du travail et l'échange ; le monde moderne a cessé d'être structuré par des valeurs partagées, un vouloir-vivre en commun et une forte solidarité. Aux relations entre personnes qui se connaissent se substitue un commerce entre des individus qui restent anonymes. L'expression la plus parfaite de ce type d'organisation se trouve dans la grande ville.

Une telle forme de construction sociale ignore les pauvres et ceux qui n'arrivent pas à tirer de l'échange de quoi vivre décemment : elle est imparfaite.

Au début du XIXe siècle, l'industriel faisait figure de héros du monde moderne, puisqu'en multipliant les emplois, il offrait aux miséreux venus des campagnes la possibilité de gagner leur vie et de s'intégrer à la nouvelle société. Cette image est bientôt remplacée par une autre : le marché du travail fixe les salaires si bas que les masses ouvrières peuvent à peine subsister. Le monde moderne ne repose pas sur une solidarité réelle. Il doit être réformé. C'est l'idée que partagent les socialistes - celle aussi qui anime les créateurs de la sociologie, Emile Durkheim en particulier.

4- Les sociétés d'ethnologues comme systèmes clos et fonctionnels

A l'origine, l'ethnologie et l'anthropologie se consacrent presque exclusivement aux sociétés premières ou aux groupes traditionnels hors d'Europe ; dans le monde industriel qui se développe sur ce continent, c'est le folklore des cellules paysannes et des milieux artisanaux qui y subsistent encore qui est étudié de la sorte.

Les ethnologues et anthropologues s'intéressent à de petites communautés. Ils ont l'impression de saisir des réalités immobiles parce que les groupes qu'ils étudient ne possèdent pas l'écriture ou ne savent pas - ou ne veulent pas - lui faire jouer un rôle important : ils ignorent l'histoire. Les chercheurs perçoivent donc les cellules qu'ils analysent comme des systèmes en équilibre ; la formation des individus y est faite pour que ceux-ci s'insèrent parfaitement dans les institutions existantes : chacun intériorise les rôles qu'il jouera par la suite ; il participera du même coup au partage des ressources et se verra reconnaître un statut, qui l'insèrera symboliquement dans le corps social. C'est l'image que donnent les ouvrages d'anthropologie sociale britannique ou ceux de certains théoriciens américains, comme Ralph Linton (1968/1936). La communauté fonctionne harmonieusement grâce à l'adéquation des rôles appris par les individus, aux statuts auxquels ils accèdent ainsi, et aux institutions qui assurent le fonctionnement de l'ensemble.

Les présupposés de l'ethnologie et de l'anthropologie sont réexaminés par ceux qui s'attachent au passage des sociétés holistes du monde traditionnel aux sociétés rationnelles et individualistes du monde moderne, à la manière de Louis Dumont $(1966$; 1977). Ils sont remis en cause par la décolonisation : pour survivre, les anthropologues sont amenés à mettre en œuvre les pratiques de terrain qu'ils avaient imaginées pour l'outre-mer colonial dans les sociétés urbanisées du monde industrialisé et urbanisé.

5- L'histoire met l'accent sur les individus exceptionnels et sur les élites

L'approche que partagent les historiens est différente. La science qu'ils pratiquaient jusqu'à la fin du XVIIIe siècle s'attachait aux princes, aux grands capitaines ou aux saints ; s'y étaient ajoutés, à partir de Voltaire, les génies qui illustraient le domaine des lettres, des arts ou des sciences.

L'histoire qui s'élabore dans les premières décennies du XIXe siècle s'inscrit en un sens dans le prolongement de celle qui se pratiquait aux siècles précédents : elle profite de la réflexion sur les sources et la manière de les lire, de les interpréter et de les conserver ; celle- 
-ci s'était développée depuis les débuts de l'humanisme et s'était affinée dans la seconde moitié du XVIIe siècle et au XVIIIe. Dans le même temps, elle s'en distingue profondément, parce qu'elle vise un autre objet : c'est à de grandes entités collectives, aux nations et aux Etats dans lesquels celles-ci s'incarnent, qu'elle s'attache désormais. Il s'agit d'objets complexes, car composés de classes qui collaborent, mais s'opposent.

L'essentiel du travail des historiens est désormais consacré aux histoires nationales (Delacroix et al., 1999) : les outils mis au point au cours de la période précédente suffisent pour traiter de leurs aspects politiques. C'est à travers des essayistes, des pamphlétaires ou des philosophes que la nation a été pensée ; c'est grâce aux hommes d'Etat qu'elle a été transformée en entité politique ; il a fallu de grands capitaines pour réunir sous la même autorité les membres longtemps dispersés des peuples et fondre dans un unité commune des groupes jusqu'alors assez dissemblables. Dans cette perspective, la grandeur des Capétiens tient à l'œuvre à laquelle ils se sont attachés : rassembler tous les Français dans une même construction politique.

Les révolutions ont fait vaciller ou disparaître les anciens régimes. Il faut désormais prendre en compte les systèmes de représentation, l'espace public où se développent les débats politiques, le jeu des élections, le travail des assemblées. Un trait demeure cependant : l'histoire que l'on écrit continue à être celle que forgent les élites - même si celles-ci sont désormais bourgeoises.

Certains refusent de mettre l'accent sur la nation. Leur ouverture sociale fait naître d'autres champs, celui de l'histoire sociale des populations rurales et des masses ouvrières d'une part, celui de l'histoire économique d'autre part. Les travaux qui leur sont consacrés explorent les sources qui font connaître ces groupes ou ces activités et permettent de suivre leur évolution. Ils montrent leur poids dans la grande histoire : ils s'intéressent pour cela aux leaders paysans, aux mouvements syndicaux, aux alliances entre partis politiques et masses sociales. L'étude historique de la société s'enrichit, mais demeurant très largement politique, elle continue à mettre l'accent sur le rôle des dirigeants.

L'histoire s'intéresse surtout aux décisions prises à la tête du corps social. Elle souligne volontiers leur rationalité, mais montre aussi qu'elles reflètent parfois le désordre des passions.

Les historiens de la fin du XIXe siècle écrivent une histoire nationale ; elle éclaire surtout le jeu des couches dominantes de la population. A la différence d'autres disciplines, elle ne réfléchit guère à ce qui fonde les sociétés dont elle retrace le destin : les nations sont pour elle des réalités premières. Lee problème est de comprendre comment celles-ci ont donné naissance à des entités politiques, et pas de savoir comment elles se sont formées.
6- L'économie repose sur l'hypothèse que les choix humains sont rationnels

Les sciences économiques reposent sur des hypothèses simples : 1- les décisions que prennent les hommes sont rationnelles, ce qui a deux conséquences : (i) on peut se dispenser de les étudier de manière empirique ; (ii) elles peuvent être mathématiquement schématisées, car elles visent à maximiser les revenus ou les satisfactions que l'on tire de la vie ; 2 - Les effets de ces décisions rationnelles ne correspondent pas toujours aux attentes de ceux qui les prennent, par suite du jeu des mécanismes qui servent à les rendre compatibles.

Ces mécanismes sont de deux types : (i) les marchés contraignent les acteurs présents dans un secteur d'activité à modifier leurs projets pour les rendre réalisables; (ii) les mécanismes globaux assurent l'ajustement de la dépense, de l'épargne et de l'investissement, et prennent en considération le jeu de la monnaie.

L'étude de ces mécanismes tient compte de la diversité des acteurs : l'économie classique distingue les individus selon le rôle qu'ils tiennent (elles distingue les propriétaires fonciers, les entrepreneurs et les salariés) et la classe à laquelle ils appartiennent; l'économie marginaliste oppose ceux qui cherchent à maximiser leur utilité (les consommateurs) et ceux qui se préoccupent de maximiser leurs revenus (les producteurs). Les variations à la marge de ces grandeurs motivent les décisions, ce qui évite les difficultés inhérentes à la définition de variables comme la valeur-travail.

La macroéconomie va plus loin dans l'analyse puisqu'elle distingue les décisions de consommation de celles de production, et prend également en compte les choix relatifs à l'épargne et ceux qui ont trait à l'investissement. Elle retrouve les agrégats sociaux qui tenaient une place essentielle dans l'économie classique, mais elle le fait d'une manière différente : son approche comptable suit les jeux de l'épargne, de l'investissement et de la création monétaire. Elle fait la différence entre les approches ex post - qui se donnent pour but d'expliquer la réalité telle qu'elle est observable après coup - et les approches ex ante - qui prennent en compte les anticipations des acteurs, y compris celles auxquelles le jeu des marchés et des ajustements globaux ne permettra pas de prendre corps.

\section{III- Les fondements des épistémologies des scien- ces de l'homme et de la société : jusqu'aux années 1960}

\section{1- Les hypothèses simplifiées de la première phase}

Dans une première phase, l'épistémologie des sciences sociales repose souvent sur des hypothèses simplificatrices : (i) l'économiste suppose que les décisions qu'il étudie sont rationnelles ; (ii) l’historien 
attribue les évolutions qu'il met en évidence aux décisions prises par un individu, monarque, président ou premier ministre, ou par les élites ; (iii) les anthropologues voient dans l'innovation un processus si rare que le progrès repose dans une large mesure sur les processus de diffusion ; (iv) les géographes sont persuadés que l'influence du milieu détermine les comportements humains.

De telles hypothèses sont si réductrices qu'elles sont rapidement critiquées. Les schémas d'explication qu'on leur substitue sont plus complexes.

\section{2- L'hypothèse fonctionnaliste ou organiciste}

Une idée s'impose : les sociétés que l'on étudie sont ainsi faites qu'elles font face à d'innombrables problèmes ; (i) certains ont trait à leur reproduction biologique (leurs membres doivent être correctement nourris ; ils doivent avoir des enfants en nombre suffisant pour remplacer les générations) ; (ii) d'autres sont relatifs à leur reproduction culturelle (les savoir-faire, les savoirs, les croyances doivent être transmis d'une génération à l'autre) ; (iii) d'autres encore concernent leur capacité et leur propension à innover (les groupes doivent imaginer de nouvelles combinaisons productives pour maîtriser plus parfaitement leur environnement) ; (iv) les derniers naissent de leur vie de relation et des techniques qui la rendent efficace.

Dernier point : toutes les sociétés que l'on peut observer fonctionnent, car dans le cas contraire, la compétition biologique et la concurrence qui se développent entre elles les auraient éliminées.

Dans ces conditions, expliquer les sociétés (petites, ce qui est la tâche des ethnologues et des anthropologues, ou grandes, ce qui incombe aux sociologues), comprendre leur devenir (ce qui revient aux historiens), analyser leur distribution spatiale et leur insertion dans l'environnement (ce qui est au cœur de la géographie), s'attacher aux jeux de pouvoir qui s'y exercent (ce qui passionne les politologues) n'implique pas l'étude de toutes les décisions. Il suffit de mettre l'accent sur celles qui assurent leur fonctionnement (Talcott Parsons, 1951). L'attention se focalise donc sur les points suivants. (i) Comment la reproduction biologique est-elle assurée? (ii) Comment la transmission culturelle est-elle réalisée? (iii) Comment la distribution de l'habitat et la circulation conduisent-elles à tirer parti de ressources dispersées et à acheminer jusqu'aux consommateurs les produits obtenus ? (iv) Comment l'ouverture à l'innovation, les crises et les révolutions confèrent-elles au corps social une plasticité qui le fait évoluer ? (v) Comment le pouvoir est-il hiérarchisé et distribué afin de résoudre les problèmes, de maintenir la stabilité ou d'assurer la réalisation d'équilibres dynamiques?

Toutes les disciplines ont mis en œuvre des explications de type fonctionnaliste. Celles-ci ont longtemps constitué l'essentiel de l'apport de l'économie.
Les historiens y ont recours lorsqu'ils soulignent le rôle des institutions et analysent les missions qui leur sont confiées. Les sociologues font de même. Dans la mesure où ils considèrent que les sociétés auxquelles ils s'attachent sont immobiles, les ethnologues ont tendance à ne voir dans les ensembles qu'ils analysent que les rouages d'une organisation totalement fonctionnelle. Les géographes imaginent la notion de genre de vie pour expliquer comment les groupes humains tirent parti de leur environnement et assurent ainsi leur reproduction biologique. Les politologues insistent sur la logique des institutions qui assurent la direction et la régulation de la vie sociale.

Une machine qui fonctionne bien n'est pas sans analogie avec un organisme sain : dans les deux cas, il $\mathrm{y}$ a des fonctions à remplir et des organes (ou des institutions) qui en ont la charge. Les sciences sociales font donc la part belle à l'organicisme - souvent mêlé au fonctionnalisme.

\section{3- De la découverte des structures au structuralisme}

Dans la réalité qu'elles explorent, les sciences sociales mettent en évidence des configurations stables. En s'attachant à la phonologie, la linguistique découvre la logique qui régit les systèmes de sons, voyelles et consonnes, et la manière dont ils se définissent les uns par rapport aux autres. Une analyse analogue peut se développer au niveau des signes - comme le montre la sémiologie. Cette orientation, ouverte par de Saussure, progresse rapidement entre les deux guerres mondiales grâce aux travaux de chercheurs russes ou à ceux de Jakobson, à Prague. La linguistique commence à faire école durant la Seconde Guerre mondiale : Jakobson est réfugié à New York. Claude Lévi-Strauss (1958) l’y rencontre et découvre ses idées. Celles-ci lui permettent d'éclairer les structures élémentaires de la parenté, puis le rôle et la signification du mythe. Avant lui, d'autres ethnologues avaient également mis en évidence des structures : Marcel Granet avait souligné les logiques de la pensée chinoise. Georges Dumézil avait mis l'accent sur l'idéologie trifonctionnelle des peuples indo-européens. En s'attachant au ressort des sociétés holistiques et à celui des sociétés individualistes, Louis Dumont $(1966$; 1977) avait ouvert une autre piste structurale en anthropologie.

L'analyse des structures fait bientôt des émules dans d'autres disciplines. Les historiens distinguent des périodes où dominent les mêmes traits - histoire ancienne, histoire médiévale, histoire moderne, etc. Ils mettent en évidence certaines logiques sociales - celles de l'esclavage ou celles de la féodalité. Les spécialistes d'histoire grecque, Moses Finley, Pierre Vernant, Pierre Vidal-Naquet ou Marcel Détienne, font comprendre le rôle du mythe dans la formation de la cité grecque et dans la naissance de la philosophie ou de la tragédie. L'histoire du Moyen Age s'ouvre également à l'histoire structurale. Dès avant guerre, Marc Bloch démonte la 
logique de la société féodale. La structure trifonctionnelle des sociétés indo-européennes s'exprime aussi dans le monde médiéval, comme le rappelle Georges Duby.

Les structures auxquelles s'attachent les historiens du monde moderne et contemporain sont différentes : elles se traduisent par la division en classes. Celle-ci est au centre de la dynamique sociale qui met fin au monde féodal et donne naissance la société industrielle moderne. Les fondements économiques de ces évolutions empêchent, un temps, de leur appliquer des approches qui ont fait leur preuve ailleurs. Ce n'est qu'assez tard, à partir des années 1950, que l'on se met à analyser les cultures de classe ; dans ce domaine, les Anglais vont plus loin que les Français, comme le montrent les études de E. Thompson et de Raymond Williams.

Depuis la fin du XIX ${ }^{e}$ siècle, les géographes sont fascinés par les structures territoriales qu'ils découvrent : régions ou structures agraires. Durant près d'un demi-siècle, elles sont au centre de leurs travaux. Elles leur paraissent d'autant plus intéressantes que les divisions qu'elles révèlent ne sont pas le fait du Prince et échappent à l'arbitraire de ses décisions : elles sont purement géographiques.

\section{4- Le rôle des masses, de leurs décisions et de la longue durée}

Des considérations normatives retardent l'emploi de la démarche scientifique dans certains domaines de la vie sociale. C'est le cas de l'étude des faits de pouvoir et de l'organisation de la vie politique ; à l'Ecole libre des Sciences politiques qu'Emile Boutmy fonde en 1881, l'enseignement repose sur deux disciplines, l'histoire et le droit constitutionnel. Le fait politique est saisi dans sa dimension événementielle (c'est le rôle de l'histoire) et dans sa dimension juridique et normative (c'est le rôle du droit constitutionnel). L'analyse des processus politiques n'apparaît qu'à la veille de la Première Guerre mondiale, et dans un domaine qui demeure marginal : le système électoral. André Siegfried, dont le Tableau de géographie politique de la France de l'Ouest (1913) ouvre la voie, se réclame d'ailleurs de la géographie, pas de la science politique. Il faut attendre les années 1930 et l'apparition, aux Etats-Unis, de la sociologie politique, pour que l'écart entre la mission affichée des institutions politiques et leur fonctionnement réel soit scientifiquement analysé.

Les préoccupations normatives sont ainsi à l'origine de véritables blocages, mais elles ne concernent qu'une partie du champ social. L'impact du parti-pris rationaliste est beaucoup plus large, car il limite le champ des investigations menées dans beaucoup de domaines. L'histoire traditionnelle soulignait à la fois la sagesse des grands hommes d'Etat, et la démesure et les excès auxquels leurs passions les conduisaient. L'histoire nationale telle qu'elle est pratiquée au XIXe siècle s'intéresse surtout aux vues à long terme, aux projets structurants, à la mise en place d'institutions efficaces. Elle insiste davantage sur la rationalité des choix effectués par les régimes modernisés que sur les excès qui peuvent parfois les accompagner. Derrière le désordre des révolutions, elle lit la mise en place de formes supérieures de la rationalité et emboîtant le pas de Hegel, elle s'intéresse aux ruses de la Raison. Il faut attendre les génocides du XXe siècle pour que des approches plus critiques s'imposent.

Quelques sciences sociales s'attachent aux décisions de tous, et pas seulement à celles des élites. C'est le cas de la géographie humaine (Claval, 2001). Le point de vue évolutionniste de ses fondateurs les conduit à s'intéresser à l'influence que le milieu exerce sur les êtres humains. Celle-ci est-elle de nature psychologique ? La curiosité que les géographes manifestent sur ce point est ancienne, puisqu'elle vient d'Hippocrate ; les enseignements de celui-ci sont repris à la Renaissance par Jean Bodin et à l'époque des Lumières par Montesquieu. Le courant n'est pas éteint dans le dernier quart du XIXe siècle, comme le prouvent certains textes de Vidal de la Blache, mais c'est finalement à un autre aspect de ces influences que la jeune discipline s'attache. La climatologie progresse alors rapidement et souligne l'impact de l'ensoleillement, de la chaleur et des précipitations sur la croissance des plantes et sur la vie des animaux. Selon les lieux, les hommes ont donc dû imaginer et mettre au point des techniques et des procédures d'exploitation différentes pour tirer leur subsistance des milieux dans lesquels ils vivaient. C'est à travers l'analyse des genres de vie ainsi constitués que la géographie française analyse les relations hommes/milieux. Cela l'affranchit des formes brutales du déterminisme et la conduit à s'intéresser aux décisions de tout un chacun, puisque tous participent à la mise en valeur de l'environnement.

Les activités des chasseurs, des pêcheurs, des collecteurs, des pasteurs ou des cultivateurs sont répétitives, puisqu'elles se reproduisent d'année en année. Le fait qu'elles appartiennent au registre de l'habitude ne les empêche pas d'être rationnelles : au cours du temps, et à travers un processus renouvelé d'initiatives et d'erreurs, des solutions satisfaisantes ont été apportées aux défis que posait chaque environnement.

La géographie humaine française ouvre ainsi une voie originale et féconde dans l'analyse de l'action humaine. Les décisions qui structurent les genres de vie sont rationnelles, même si elles n'ont pas été inventées d'un coup et résultent de multiples essais - ce qui attire l'attention sur le rôle de la longue durée dans la construction des savoir-faire collectifs. Ceux-ci ne sont pas nés de l'initiative d'élites étroites. Ils résultent généralement de l'imagination de gens modestes. L'attention se porte ainsi sur le rôle des masses, de ceux qui ne savent ni lire, ni écrire, ne laissent guère de trace de leur existence, mais doivent faire face aux difficultés de la vie de tous les jours et essaient par tous les moyens d'y 
faire face. La géographie humaine propose ainsi plus précocement que les autres sciences humaines une démarche qui s'attache au rôle des petites gens - une démarche plus 'démocratique', en un sens.

L'attitude de l'ethnologie et celle de la sociologie sont au départ très différentes. Ces disciplines sont loin de considérer les décisions des groupes qu'elles étudient comme rationnelles : les ethnologues parlent de mentalités prélogiques ; ils s'attachent à la pensée magique, s'étonnent de l'existence de tabous, détaillent les préjugés dominants. Le tableau qu'ils dressent des sociétés primitives et traditionnelles est très critique.

Les attitudes changent à partir du moment où l'ethnologie devient une science de terrain : à la suite de l'expérience de Bronislaw Malinowski aux îles Trobriand, on considère que le chercheur doit longuement séjourner longuement dans les groupes qu'il étudie, apprendre leur langue et analyser toutes les facettes de leurs comportements. Les anthropologues sont ainsi amenés à faire une place importante aux bases matérielles de l'existence des groupes avec lesquels ils cohabitent, à leurs techniques de chasse et de pêche, et aux plantes qu'ils connaissent et qu'ils ont appris à collecter ou à cultiver. Ils s'intéressent aux champs, aux jardins et aux récoltes qu'ils portent, ainsi qu'aux pâturages et aux troupeaux qu'ils nourrissent. Comme les géographes, dont ils empruntent souvent les méthodes, ils décrivent des genres de vie et soulignent l'ingéniosité des choix sur lesquels ceux-ci reposent.

Certains historiens adoptent également ces démarches. L'histoire sociale, celle du monde ouvrier en particulier, auquel on s'intéresse depuis les années 1860, met l'accent sur les composantes populaires des sociétés modernes. L'histoire économique s'attache au jeu des marchés et le mouvement des prix ; elle découvre des cycles dont la périodicité est diverse. Elle se détourne ainsi des perspectives élitistes qui dominaient encore l'histoire événementielle même après que celle-ci ait pris le tournant national.

Lucien Febvre comprend ce que la géographie humaine apporte par son analyse des petites décisions et de la rationalité qui les caractérise. Marc Bloch partage cette sensibilité aux acquis de la géographie humaine ; il s'intéresse aussi à l'histoire économique. En fondant l'Ecole des Annales, ces deux historiens attirent l'attention sur l'écoulement du temps et la longue durée, dont Fernand Braudel se fera bientôt le théoricien (Couteau-Bégarié, 1983).

\section{5- Le rôle des représentations et des imaginaires : la fon- dation du social}

Une autre interprétation des sciences de l'homme et de la société se constitue dans certaines disciplines à la fin du XIXe siècle et dans les premières décennies du XXe. Elle joue un rôle essentiel dans l'ethnologie française et en histoire, avec l'étude des représentations et des mentalités. Elle s'esquisse également dans d'autres disciplines.

L'essor de l'ethnologie française est préparé au début du XXe siècle par Emile Durkheim, très attentif aux recherches des anthropologues de langue anglaise, comme le montrent Les Formes élémentaires de la vie religieuse : le système totémique en Australie (1912). L'ethnologie française se caractérise alors par sa dimension réflexive. Il y a une raison simple à cela : rien ne permet de financer des recherches de terrain aux colonies. Celles-ci ne sont ouvertes qu'aux missionnaires, Leenhardt en Nouvelle-Calédonie par exemple. L'orientation réflexive de l'ethnologie française se maintient entre les deux guerres, à l'exemple de Marcel Mauss, mais l'Afrique s'ouvre aux investigations directes, comme en témoigne la mission Dakar-Djibouti organisée par Marcel Griaule en 1931-1932 (Clifford, 1988).

Les travaux menés en France prennent une orientation différente de celle qui prévaut alors en Grande-Bretagne et aux Etats-Unis. Les ethnologues français ne s'attachent guère aux bases matérielles de la vie des groupes, à la manière dont ils cultivent la terre et élèvent le bétail, ou à leur habitat et à leur équipement domestique. Ils se passionnent en revanche pour les croyances religieuses et les rituels qui leur sont associés. Ils analysent longuement les fêtes, les cérémonies, les masques ou le culte des morts. Plusieurs raisons les y poussent : ils cherchent à cerner le lien social, qui s'exprime, pensent-ils, à ces occasions: la fascination pour l'art nègre, qu'accentue le surréalisme, oriente leur quête vers les ressorts les plus profonds de la vie humaine, comme on le sent chez Michel Leiris ; les mythes, dont Marcel Griaule mène la collecte chez les Dogons et dont Roger Caillois explore la logique, révèlent les croyances fondamentales ; masques et statues font connaître les imaginaires des groupes, et donnent une idée des rêves et des inquiétudes qui les habitent.

Ce qui est au cœur de ces ethnographies, c'est ce qui fonde les sociétés, et pas la manière dont elles fonctionnent.

Des ouvertures analogues se produisent en histoire. On savait depuis Droysen (1855), dans les années 1860, que la discipline cherchait davantage à comprendre les trajectoires qu'elle reconstituait qu'à les expliquer. En France, l'éclosion de curiosités analogues se produit entre les deux guerres mondiales. En s'attachant aux Rois thaumaturges, Marc Bloch explore l'ensemble des croyances qui donnent à la monarchie française d'Ancien Régime sa singularité. Le thème est repris et amplifié trente ans plus tard par Ernst Kantorowics, dans sa célèbre étude sur Les Deux Corps du Roi (1957) : il y oppose le corps mortel du roi à son corps souverain, que rien ne peut faire disparaître, ce qui assure la continuité monarchique et politique.

En analysant Rabelais et le problème de l'incroyance au XVIe siècle, Lucien Febvre ouvre une voie parallèle : il ne s'attache pas aux imaginaires fondateurs des régimes politiques et des sociétés, mais 
aux mentalités qui caractérisent certaines époques et certains milieux.

L'Ecole des Annales, où s'opère en France l'ouverture aux préoccupations nouvelles, explore simultanément trois pistes (Couteau-Bégarié, 1983) : celle de l'histoire économique et des cycles, celle de la rationalité des petites décisions et de leur impact sur la longue durée, et celle des imaginaires et des identités. Comme chez les ethnologues, l'exploration des imaginaires met en évidence les fondations sur lesquelles reposent sociétés et régimes politiques.

\section{6- Des praticiens qui cherchent une caution dans les épis- témologies positivistes ou néo-positivistes}

A la fin du XIX ${ }^{\mathrm{e}}$ siècle et dans la première moitié du $\mathrm{XX}^{\mathrm{e}}$, ceux qui développent les sciences sociales sont rarement des théoriciens. Ce qu'ils inventent, c'est un métier : il apprennent quelles sources et quels documents utiliser et quelles enquêtes mener ; ils définissent les procédures à suivre pour les exploiter et en tirer des tableaux ou des récits. Ces derniers soulignent la juxtaposition de faits intéressants ou leur enchaînement dans des séquences causales.

Des doutes assaillent souvent ces pionniers ou leurs successeurs immédiats. Ils cherchent alors une caution - et la trouvent chez les philosophes ou les représentants des sciences physiques ou naturelles. A la fin du XIX $\mathrm{X}^{\mathrm{e}}$ siècle, la vision dominante est positiviste. La réalité peut se décomposer en faits qu'il est possible de décrire de manière objective et dans bien des cas, de mesurer. La tâche du chercheur est double : collecter des données et déceler, grâce à l'observation ou à l'expérience, les relations causales qui existent entre eux. Le progrès implique une longue patience et repose sur l'accumulation indéfinie des faits et des liaisons qui existent entre eux.

Les présupposés du positivisme s'appliquent difficilement à certains aspects des sciences sociales : l'observation ne donne pas accès aux processus mentaux qui sont à l'origine des décisions que prennent les acteurs ; enquêtes et entrevues tournent la difficulté, mais sans que leurs résultats aient une objectivité égale à celle qui caractérise les sciences physiques ou naturelles. Malgré cela, de multiples efforts sont faits pour calquer les approches de la société sur celles de la physique ou des sciences naturelles. Lorsque Jean Brunhes (1910) écrit, sous le titre La Géographie humaine, le premier traité qui précise, en langue française, les traits de cette nouvelle discipline, il lui donne comme sous-titre : Essai de classification positive. L'ouvrage énumère aussi objectivement que possible les 'faits essentiels' de la discipline. Il distingue (i) les faits d'occupation improductive du sol, (ii) les faits de conquête végétale et animale et (iii) les faits d'occupation destructive (dévastations végétales et animales, exploitations minérales). L'étape suivante consiste, à travers des monographies régionales, à poursuivre « l'étude synthétique des connexions réelles entre les faits essentiels ». A côté de faits essentiels (la route par exemple), ce travail met en évidence, des faits accessoires (les moyens de transport). Ceux-ci « semblent comme envelopper et vêtir les faits matériels, de la même manière que les vêtements accompagnent et couvrent la réalité vivante des corps vivants » (p. 265). Ils méritent l'attention, mais ne sont pas au cœur du travail.

Pierre Deffontaines est fidèle aux enseignements de Jean Brunhes. Le parti-pris positif qu'il en reçoit le contraint à des choix curieux, en géographie religieuse par exemple.

"Le géographe est [...] appelé à conserver à l'égard des faits religieux une attitude de pur observateur, ne cherchant pas à étudier le fondement, l'origine ou l'évolution des systèmes religieux et la valeur respective de ceux-ci. Il se borne à noter les répercussions géographiques des faits de religion sur le paysage, il réduit ainsi le point de vue religieux à des éléments extérieurs et physionomiques, laissant délibérément de côté le domaine majeur de la vie intérieure" (Deffontaines, 1966, p. 17-18).

On comprend à cet exemple les critiques que suscite la conception positive de la science. Les idées évoluent donc. Les physiciens imaginent, entre les deux guerres mondiales, une version épurée du positivisme, dont le cercle de Vienne fournit la formulation la plus claire. Avec l'arrivée du nazisme, ses membres se dispersent: durant une génération, le néo-positivisme logique qu'ils prônent domine la réflexion épistémologique. Il est beaucoup plus satisfaisant que les conceptions qu'il remplace, car il reconnaît le rôle de l'imagination dans la construction des hypothèses ; l'expérience élimine alors celles qui ne rendent pas compte des faits. Pour les néo-positivistes, la démarche qu'ils théorisent est également valable pour les sciences de la matière, pour celles de la vie et pour celles de l'homme et de la société. Ils condamnent 'l'exceptionnalisme' de ces dernières - la nécessité qu'elles invoquent de suivre d'autres voies parce qu'elles traitent d'un sujet différent.

Les progrès que connaissent à la même époque les mathématiques appliquées et la statistique contribuent au succès du néo-positivisme logique. Beaucoup de chercheurs s'en réclament : des ethnologues comme Lévi-Strauss, des sociologues, des politologues. Les idées du cercle de Vienne donnent à la Nouvelle Géographie une base épistémologique qui contribue largement à son succès.

Le néo-positivisme logique conduit à une certaine unification des sciences de l'homme et de la société, mais celle-ci est réalisée de l'extérieur. Un mouvement parallèle et plus profond œuvre à leur rapprochement, mais de l'intérieur cette fois.

\section{7- De l'impérialisme intellectuel de certaines sciences de} l'homme à l'idée de pluridisciplinarité 
La société est si complexe et ses manifestations sont si diverses qu'elle est étudiée à partir de perspectives variées bien plutôt que comme un objet unique. La linguistique l'aborde à travers les langues parlées dans le monde d'aujourd'hui ou d'hier, l'histoire à travers les documents qui témoignent du passé, la géographie à travers la distribution des groupes humains et les marques qu'ils laissent dans le paysage, la préhistoire et l'ethnographie à travers les artefacts dont les hommes se sont entourés, ou s'entourent, le folklore à travers les activités vernaculaires et les traditions orales du monde traditionnel, et les sciences politiques à travers les institutions du pouvoir et les lieux où il s'incarne.

La sociologie est la seule discipline qui aborde de front le fait social : dès Auguste Comte, elle a l'ambition de se substituer aux sciences qui l'ont précédée ou se développent parallèlement à elle. A la fin du XIX ${ }^{\mathrm{e}}$ siècle, elle traite encore de toutes les sociétés, celles d'aujourd'hui et celles du passé, comme le montrent l'œuvre d'Emile Durkheim en France ou de Max Weber en Allemagne. Ce n'est que progressivement qu'elle se tourne plutôt vers les sociétés contemporaines, pour laquelle elle a mis au point des techniques d'enquête efficaces.

Dans le sillage de Durkheim, l'impérialisme intellectuel de la sociologie s'affirme aux alentours de 1900. Il est vigoureusement critiqué par la géographie et par l'histoire (par Lucien Febvre en particulier) entre le début du XX $\mathrm{XX}^{\mathrm{e}}$ siècle et la Seconde Guerre mondiale. A la même époque, certains historiens, Marc Bloch en particulier, considèrent que leur discipline est au fondement même du social et revendiquent pour elle une position privilégiée.

Les attitudes changent après la Seconde Guerre mondiale. Les chercheurs soulignent que les disciplines qui traitent de la vie collective diffèrent par les perspectives qu'elles retiennent, les documents qu'elles exploitent et les méthodes qu'elles emploient, mais ils reconnaissent que leur finalité est la même : éclairer l'homme comme être social et expliquer la diversité, la logique et le fonctionnement des groupes qu'il crée et des institutions qui les structurent. Pour éclairer les problèmes que rencontre telle ou telle science, les points de vue développés par les autres disciplines se révèlent souvent féconds car ils permettent de surmonter des blocages et de faire surgir de nouvelles questions et de nouvelles façons de les étudier.

Ce rapprochement des sciences de l'homme social est salué comme un progrès majeur : on se met à parler de pluridisciplinarité. Celle-ci permet d'explorer les angles morts qui subsistent entre les diverses sciences de l'homme - d'approfondir l'histoire économique, en conjuguant les leçons de l'histoire et celles de l'économie, ou la géographie sociale en combinant les approches de la géographie et de la sociologie. La pluridisciplinarité souligne ainsi ce qu'apportent, dans un champ d'étude, des théories importées d'autres domaines.

La réflexion sur la pluridisciplinarité témoigne d'une prise de conscience : celle de la proximité de toutes les recherches qui portent sur l'homme social et sur la vie en société. Mais ce rapprochement demeure limité : il ne débouche pas sur une réflexion sur le noyau commun à toutes ces approches.

\section{IV- Epistémologie, sciences sociales et géographie : à partir des années 1970}

L'étude de la société se transforme profondément à la fin des années 1960 et au cours des années 1970 . Le rationalisme et la science qu'il a engendrée ne jouissent plus du même prestige. Deux facteurs concourent à cette évolution. (i) Le progrès des technologies de destruction massive conduit aux horreurs de la guerre moderne et à la menace que font peser les armes nucléaires ou chimiques. (ii) On prend conscience du lien qui existe entre l'universalisme rationaliste dont l'Occident se réclame depuis la Renaissance, l'impérialisme qu'il a pratiqué et la désintégration de beaucoup des sociétés et des cultures qu'il a côtoyées.

L'idée que l'on se faisait de l'intelligence est en procès. La réflexion épistémologique ne se développe plus dans le même contexte.

\section{1- L'épistémologie comme entreprise de déconstruction.}

La critique philosophique du rationalisme s'organise au XIX ${ }^{\mathrm{e}}$ siècle. Elle s'exprime alors dans les œuvres de Schopenhauer et de Nietzsche. Le premier distingue le monde de la représentation, qui est celui du nettement délimité, du rationnel et du sensé (Ferry et Capelier, 2014, p. 320) et le monde de la volonté, qui est celui des forces aveugles, des pulsions profondes et des instincts : un ensemble inconscient et dénué de sens (ibid., p. 323). Le monde de la représentation est rassurant, mais ce n'est pas lui qui modèle la réalité. Celle-ci est configurée par l'ensemble des forces subsumées sous le terme de volonté.

Le vocabulaire mis en œuvre par Nietzsche est différent. Il parle de volonté de puissance, ou plus simplement de vie, là où Schopenhauer mettait en avant la volonté (ibid., p. 323). Il critique radicalement "tous les idéaux, quels qu'ils soient, métaphysiques, religieux ou politiques, Dieu, le progrès, la démocratie, la révolution, le socialisme, les Droits de l'homme, la science, la nature, la république, etc." (ibid., p. 350), et désigne "sous le terme de 'nihilisme' toutes les pensées de l'idéal opposé au réel'” (ibid., p. 351).

Le problème de Nietzche est d'en finir avec les idéaux. Pour ce faire :

"il ne suffit pas de leur opposer les arguments de la raison, il faut encore, et surtout, dévoiler leurs motivations cachées au plus profond, lesquelles tiennent à la volonté de nier la vie ; il faut donc refuser de se placer sur le terrain de discussion que proposent les idéalistes et chercher plutôt, sous sa 
surface, la haine de la vie qui les anime, la guerre qu'ils lui livrent et contre laquelle la seule réponse à la hauteur est de leur faire la guerre à notre tour" (ibid., p. 352).

Comment mener cette contre-offensive? En retraçant la généalogie des idoles :

"[...] l'anti-théorie de la connaissance que constitue la démarche généalogique [...] présente un double visage : d'un côté, elle mène une critique radicale de toutes les certitudes, dynamitant par exemple la conviction que notre conscience est transparente à elle-même, que les vérités objectives de la science méritent qu'on s'y soumette parce qu'elles sont au-dessus des passions humaines, que nos valeurs morales doivent s'imposer à tous parce qu'elles sont universelles ; de l'autre côté, elle ouvre une nouvelle dimension de la connaissance qui consiste, en quelque sorte, à explorer le sous-sol de nos représentations pour en découvrir les motivations profondes, liées à nos pulsions et à nos stratégies inconscientes destinées à légitimer notre rapport, conquérant et craintif, à la vie" (ibid., p. 357358)

Nietzsche initie les opérations de déconstruction ; il les mène en termes de généalogie et remonte à l'origine des croyances. Il n'est pas le seul à opérer de la sorte : Marx s'attache aux processus cachés qui prennent place au sein de l'économie ; Freud analyse l'inconscient psychique. La critique de Nietzsche s'en prend plutôt à la religion, à la morale et à la philosophie. Elle est moins directement focalisée sur la démarche scientifique. L'idée de déconstruire celle-ci ne se précise que plus tard. Elle ne prend pour cible les sciences de l'homme et de la société que lorsque celles-ci affirment leur autorité, au milieu du XX $\mathrm{X}^{\mathrm{e}}$ siècle. Le mouvement, animé par Michel Foucault, François Lyotard, Jacques Derrida ou Gilles Deleuze, prend rapidement de l'ampleur (Cusset, 1983).

Foucault est représentatif de cette génération. Pour lui, c'est à la fin du XVIII ${ }^{\mathrm{e}}$ siècle et au début du XIX ${ }^{\mathrm{e}}$ que pour "la première fois depuis qu'il existe des êtres humains et qui vivent en société, l'homme, isolé ou en groupe, [est] devenu objet de science : c'est un événement dans l'ordre du savoir" (Foucault, 1965, p. 356).

Et Foucault continue :

"Et cet événement s'est lui-même produit dans une redistribution générale de l'épistémè : lorsque, quittant l'espace de la représentation, les êtres vivants se sont logés dans la profondeur spécifique de la vie, les richesses dans la poussée progressive des formes de production, les mots dans le devenir des langages. Il était bien nécessaire dans ces conditions que la connaissance de l'homme apparaisse, en sa visée scientifique, comme contemporaine et de même grain que la biologie, l'économie et la philologie, si bien qu'on a vu en elle un des progrès les plus décisifs faits, dans l'histoire de la culture européenne, par la rationalité empirique" (ibid., p. 356).

Pour Foucault, les sciences humaines sont nées dans le trièdre constitué par trois des nouveaux savoirs scientifiques qui se constituent au tournant des XVIII ${ }^{\mathrm{e}}$ et XIX ${ }^{\mathrm{e}}$ siècle, la biologie, l'économie et la linguistique (ces deux dernières disciplines n'étant pas, pour lui, des disciplines de l'homme et de la société). Les sciences de l'homme ne méritent pas le titre qu'elles revendiquent : Foucault précise, “ce mot même [de sciences] est peut-être trop fort : disons pour être plus neutre encore, cet ensemble de discours" (ibid., p. 355).

Quel est alors leur statut? Foucault l'analyse ainsi :

“De ce trièdre épistémologique [biologie, économie, linguistique], les sciences humaines sont exclues [...]. Mais on peut dire aussi qu'elles sont incluses par lui, car c'est dans l'interstice de ces savoirs, plus exactement dans le volume défini par leurs trois dimensions, qu'elles trouvent leur place. Cette situation [...] les met en rapport avec toutes les autres formes de savoir [...] ; elles ont le projet [...] de se donner une formalisation mathématique ; elles procèdent selon des modèles ou des concepts qu'elles ont emprunté à la biologie, à l'économie ou aux sciences du langage; elles s'adressent enfin à ce mode d'être de l'homme que la philosophie cherche à penser au niveau de la finitude radicale, tandis qu'elles-mêmes veulent en parcourir les manifestations empiriques" (ibid., p. 358).

Les discours sur l'homme en société que tiennent les sciences sociales sont ainsi déconstruits : parce qu'inscrits dans le trièdre de trois disciplines qui explorent l'inconscient de la vie, de l'économie et du langage, ces champs sont "voués à une instabilité essentielle" :

"Ce qui explique la difficulté des 'sciences humaines', leur précarité, leur incertitude comme sciences, leur dangereuse familiarité avec la philosophie, leur appui mal défini sur d'autres domaines du savoir, leur caractère toujours second et dérivé, mais leur prétention à l'universel, ce n'est pas, comme on le dit souvent, l'extrême densité de leur objet ; ce n'est pas le statut métaphysique, ou l'ineffaçable transcendance de cet homme dont elles parlent, mais bien la complexité de la configuration épistémologique où elles se trouvent placées, leur rapport constant aux trois dimensions qui leur donnent leur espace" (ibid, p. 359).

Et Foucault de conclure :

"En effet parmi toutes les mutations qui ont affecté le savoir des choses et de leur ordre, $[. .$.$] , une seule, celle qui a commen-$ cé il y a un siècle et demi et qui peut-être est en train de se clore, a laissé apparaître la figure de l'homme. Et ce n'était point là libération d'une vieille inquiétude, passage à la conscience lumineuse d'un souci mil- 
lénaire, accès à l'objectivité de ce qui longtemps était resté pris dans des croyances ou des philosophies : c'était l'effet d'un changement dans les dispositions fondamentales du savoir. L'homme est une invention dont l'archéologie de notre pensée montre aisément la date récente. Et peut-être la fin prochaine" (ibid., p. 398).

Les sciences de l'homme et de la société ne sont guère que des discours qu'il faut étudier dans la même perspective que les autres discours - ce à quoi Foucault s'emploiera dans L'Archéologie du savoir (1969). Il montrera ensuite (Surveiller et punir, 1976) l'association qui se tisse au XVIII ${ }^{\mathrm{e}}$ siècle (surtout) entre les deux usages du regard : source de savoir, il est aussi un outil de surveillance indispensable à qui veut discipliner l'homme.

Dans cette entreprise générale de déconstruction, Lyotard, Derrida et Deleuze explorent d'autres pistes. Ce dernier s'attache par exemple au foisonnement des rhizomes, ces enracinements multiples qui permettent aux constructions sociales d'échapper à la tyrannie unificatrice du regard ; leurs racines parlent d'un univers qui résiste à toute centralisation.

Présentée d'une autre façon, la déconstruction des sciences sociales souligne qu'une partie essentielle de leurs prétendus résultats est faite de récits dont rien ne garantit la validité, mais qui fournissent une interprétation de l'évolution de la société ou du fonctionnement de leurs institutions. Lyotard (1979) montre la vanité de ces discours. Dans le domaine scientifique, une des taches auxquelles la postmodernité doit s'atteler, c'est de débarrasser les savoirs sur l'homme et la société de toutes les scories que les grands récits y ont laissées. Toute narration d'ensemble cohérente devient suspecte : l'explication qu'elle prétend apporter ne repose pas sur des preuves solides ; ce n'est, le plus souvent, qu'une construction idéologique. La critique vaut en particulier pour l'histoire, très friande de grands récits qui racontent la construction de la nation, du territoire national ou de la République.

La déconstruction s'inscrit dans un mouvement philosophique initié au XIX ${ }^{\mathrm{e}}$ siècle et dont le champ s'élargit progressivement aux sciences de l'homme social et de la société dans la seconde moitié du $\mathrm{XX}^{\mathrm{e}}$ siècle. Aux techniques de l'archéologie des savoirs, que mettent en œuvre les philosophes, s'ajoutent celles qui soulignent le rôle que jouent les mythes fondateurs auxquels les créateurs des sciences sociales ont souvent fait appel. Le travail de déconstruction échappe alors aux philosophes ; il s'inspire de la réflexion sur la construction et l'interprétation du mythe qui se développe de Roger Caillois à Claude Lévi-Strauss et à Paul Ricœur (1985). Ce qui est reproché à Hobbes, à Locke ou à Rousseau, c'est d'évoquer des évènements survenus dans un passé très lointain, à des époques dont nous ne savons rien (Claval, 1980). Les narrations ainsi proposées ressemblent à celles que le mythe offrait dans les sociétés premières. Elles ne se rapportent pas au temps réel de l'histoire que nous vivons, mais à une du- rée préhistorique assez proche du temps du rêve des mythologies. Si l'on veut rendre les sciences sociales plus rigoureuses, il convient de mettre en évidence le rôle que les courts récits par lesquelles elles s'ouvrent souvent ont joué, et continuent à jouer, dans la construction des idéologies du monde moderne.

La dénonciation des grands récits devient ainsi un des leitmotive de la déconstruction.

\section{2- L'impact des philosophies de l'inconscient}

Dans leurs premiers développements, les sciences sociales mettaient l'accent sur l'existence de déterminations qui expliquaient l'enchaînement des phénomènes et se fondaient sur une logique des ensembles et de l'identité (la logique du tiers exclu, pour faire simple). Pour elles, la raison était au fondement de la vie sociale : c'était ce que soutenait Hobbes dans sa théorie du contrat social, puisque celui-ci n'était signé que pour mettre fin à la guerre de tous contre tous ; c'était le cas des approches fonctionnalistes, puisqu'elles montraient à quelles conditions une société pouvait s'épanouir et apporter des avantages réels à ses membres. La critique des épistémologies du contrat social se développe dès le début du XIX ${ }^{\mathrm{e}}$ siècle. Les justifications fonctionnalistes ou organicistes du vivre ensemble ne sont plus aussi convaincantes maintenant que l'on sait qu'au sein de beaucoup de groupes humains, les solidarités ne sont pas manifestes.

D'autres interprétations se dessinent durant l'entre-deux-guerres. Elles trouvent leur expression achevée à partir des années 1960. Souvent inspirées au départ par le surréalisme et la psychanalyse, elles prennent en compte le jeu des imaginaires (comme chez Castoriadis), ou s'attachent à la forme particulière de ceux-ci que constitue l'utopie (comme chez Henri Lefebvre). A leur sujet, on parle souvent, d'interactionnisme symbolique.

Pour Castoriadis (1975), la société ne s'explique pas par un jeu de déterminations. Au moins au départ, c'est une création de l'imaginaire collectif. La géographie classique, la géographie néo-positiviste et la géographie marxiste ont en commun de s'appuyer sur la logique des déterminations. Dans certains cas en revanche, la réflexion sur le territoire met en jeu la logique de la création.

L'identification au groupe et au territoire n'est pas parachutée depuis les hautes couches de la société ; c'est une expérience première des hommes. Pour l'expliquer, Castoriadis fait appel à l'imaginaire - non pas l'imaginaire vulgaire que véhiculent les médias, mais un imaginaire qui naît au tréfonds de chaque être et témoigne de son pouvoir de création (sur cet aspect de Castoriadis, voir da Silva, 2013). Castoriadis est, entre autres, psychanalyste : pour lui, la vérité de l'homme ne se situe pas seulement - ou pas essentiellement - dans sa raison, mais dans le jaillissement renouvelé qui se produit en lui. En cela, Castoriadis 
approfondit le courant de réflexion qui, dans les premières décennies du XX $\mathrm{X}^{\mathrm{e}}$ siècle, proposait une interprétation du social qui n'était pas fonctionnelle et trouvait sa source dans la dimension symbolique de la nature humaine. Cette orientation était sensible chez certains anthropologues, Caillois par exemple. Les recherches de l'ethnologie française de l'entre-deux-guerres, celles de Michel Leiris ou de Marcel Griaule, s'inscrivaient aussi dans cette direction (Clifford, 1988).

Pour cette perspective, l'agenda des sciences de l'homme social cesse de se limiter à l'analyse fonctionnelle des sociétés, qu'il s'agisse de leur reproduction biologique et culturelle ou de l'organisation institutionnelle qui leur permet de satisfaire leurs besoins matériels, de résoudre leurs conflits et de faire face aux agressions extérieures. L'étude des représentations, de la pensée symbolique et de toutes les manifestations qui donnent un sens à la collective passe au premier plan.

\section{3- De l'idée de révolution scientifique à celle de tournant disciplinaire}

Les recherches consacrées à l'histoire de la physique se multiplient à partir de la fin du XIX ${ }^{\mathrm{e}}$ siècle : c'est à leur occasion que l'épistémologie prend son visage moderne. On y suit pas à pas les avancées réalisées dans l'explication du mouvement ou de la lumière. La Raison sort de sa superbe : elle ne domine plus la scène scientifique ; elle accompagne les progrès de la recherche, se nourrit de ses leçons et s'affranchit des errements de la pensée vulgaire à travers des ruptures épistémologiques, comme le souligne Gaston Bachelard (1934 ; 1938). Alexandre Koyré (1966) décrit la mutation que Galilée fait subir à la physique, et qui condamne les hypothèses sur lesquelles reposait celle d'Aristote. Au $\mathrm{XX}^{\mathrm{e}}$ siècle, les transformations que subit l'étude de la lumière sont du même ordre : la théorie ondulatoire est remise en doute par l'approche corpusculaire, seule capable de rendre compte des observations qui se multiplient alors. La révolution s'achève lorsque s'impose l'idée de la double nature du rayon lumineux : corpusculaire, avec le photon, et ondulatoire dans le sillage qu'il laisse.

C'est de ces exemples que Thomas Kuhn s'inspire lorsqu'il propose en 1962 sa théorie des révolutions scientifiques. Le succès de l'ouvrage est foudroyant. Dans les sciences sociales, il sert à souligner la signification des mutations qu'elles traversent. Les tenants de la Nouvelle Géographie insistent ainsi sur les ruptures dont est née cette manière jusque-là inédite de concevoir la discipline : rupture avec le propos de base de la géographie humaine de la fin du XIX ${ }^{\mathrm{e}}$ siècle, puisque c'est du rôle de la distance dans la vie sociale que l'on traite, et plus des relations hommes/milieux ; rupture des démarches mobilisées aussi, puisque l'exceptionnalisme qui était de mode dans la première moitié du XX $\mathrm{XX}^{\mathrm{e}}$ siècle est récusé, et que la discipline fait désormais largement appel à l'outil mathématique et statistique.

L'idée de révolution scientifique a d'autant plus de succès qu'elle conduit à distinguer deux niveaux de recherche : (i) celui des besogneux qui accumulent observations et résultats sans être capables d'adapter l'outil théorique à ce qu'elles apportent de neuf ; (ii) celui des imaginatifs qui formulent à un niveau supérieur des hypothèses et créent de nouveaux outils théoriques ; ce sont eux qui forgent les nouveaux paradigmes.

La mise en évidence de ces deux niveaux a un effet puissant : plus personne ne veut appartenir à la piétaille de la science. Ce qui compte, c'est d'initier des révolutions scientifiques. Celles-ci se multiplient donc rapidement! En physique trois siècles s'étaient écoulés entre la révolution galiléenne et la révolution quantique. Dans les sciences de l'homme et de la société, les révolutions se bousculent : en quelques années on voit déferler en géographie une vague phénoménologique associée à la nouvelle curiosité pour l'esprit des lieux, et une poussée radicale et critique qui récuse tout ce qui l'a précédé. Dès le milieu des années 1970, il apparaît que l'idée de révolution scientifique est galvaudée lorsqu'elle est appliquée aux sciences de l'homme et de la société.

Celles-ci changent évidemment, mais sans connaître de mutations fondamentales de leur appareil conceptuel et de leurs méthodes d'analyse. Ce qui se produit est différent : c'est l'angle sous lequel leur domaine est abordé qui n'est plus le même. L'histoire connaît ainsi un tournant linguistique, qui tient à l'attention accrue accordée à l'évolution des mots et des conceptions qui leur sont attachées ; la sociologie passe par un tournant spatial, qui la conduit à prendre en compte la dimension matérielle des réalités qu'elle analyse, leur étendue, leur permanence ou leur labilité - et à remettre ainsi en cause l'idée de société comme réalité première. Quelques années plus tard, les géographes prennent conscience du tournant culturel que leur discipline traverse depuis les années 1970, et qui les conduit à accorder plus d'attention à la diversité des groupes humains, à leurs projets, aux marques qu'ils impriment dans le paysage et aux idées qui les animent.

Si les sciences de l'homme et de la société se transforment, ce n'est pas parce qu'elles connaissent des révolutions au sens kuhnien du terme, c'est parce que ceux qui les pratiquent n'envisagent plus de la même manière la réalité dont ils traitent. Entre la géographie classique et la nouvelle géographie, il y a glissement de l'attention : focalisée au départ sur l'influence que le milieu exerce sur l'homme, elle se dirige désormais vers l'appréhension de tout ce qui, dans l'organisation des sociétés humaines, reflète le jeu de la distance, de la proximité ou de l'éloignement. Jusqu'aux années 1970, les géographes abordent l'analyse de la dimension spatiale des mécanismes sociaux et économiques en utilisant les outils que leur offraient l'économie, la sociologie ou l'anthropologie. Ces outils étaient adap- 
tés à des approches fonctionnalistes et considéraient les sociétés comme des machines dont il fallait expliquer la marche. Ce que les tournants que nous venons d'évoquer apportent, c'est l'idée que le modèle mécaniste négligeait certaines des composantes essentielles de la vie sociale : celle-ci ne peut se comprendre si on ignore la subjectivité des décisions humaines et le rôle du symbolisme et des imaginaires dont sont porteurs les individus et les groupes.

L'idée de tournant définit donc les modalités particulières de l'évolution de savoirs relatifs à la société et à la culture.

\section{4- Le tournant praxéologique}

Tout le monde est conscient du tournant culturel que vient de traverser la géographie. On parle moins du tournant praxéologique qui affecte l'ensemble des sciences sociales (Montbrial, 2002 ; 2006). Au XIX ${ }^{\mathrm{e}}$ siècle et dans la première moitié du $\mathrm{XX}$, les chercheurs de ces disciplines prenaient modèle sur les sciences de la matière ou de la nature. Ces dernières expliquaient la situation à l'instant $(\mathrm{t})$ par le jeu de forces présentes à l'instant ( $\mathrm{t}-1)$, ou, dans le cas des mécanismes de rétroaction, par la mise en jeu quasi-instantanée de mécanismes de rétroaction (donc $\mathrm{t}-\varepsilon$ ). Dans ce contexte, et pour expliquer les situations actuelles, les sciences sociales mettaient naturellement l'accent sur le poids des héritages et le jeu des contraintes qui leur étaient imposées de l'extérieur.

Un des traits essentiels des réalités sociales était négligé : l'état que revêtira la société à l'instant $(t+1)$ ne dépend pas seulement de l'état des forces à l'instant ( $t$ ). Il résulte de décisions prises par des acteurs dont les choix sont dictés à la fois par leur analyse de la situation à l'instant $(\mathrm{t})$ et par l'idée qu'ils se font de ce qu'elle doit être dans le futur (c'est-à-dire au temps $t+1$ ). Au moment du choix, les images que l'on se fait du futur entrent en compte.

Le tournant praxéologique que connaissent aujourd'hui les sciences sociales vient de là : le monde social - et la géographie par laquelle il s'exprime dans l'espace - résultent des décisions d'une pluralités d'acteurs. Au moment où ils les préparent, chacun de ceux-ci aimerait disposer d'une information parfaite sur la situation dans laquelle il se trouve, mais les moyens dont il dispose pour l'acquérir sont limités d'autant que beaucoup de ses partenaires lui cachent leur jeu. Chacun prend à la fois en compte le tableau nécessairement imparfait qu'il se fait de la situation, et l'image du futur auquel il aspire.

Le monde tel qu'il est ne résulte pas de choix faits par un décideur unique qui, à l'instar de Dieu le Père, serait omniscient et tout-puissant. Il est le produit d'une multitude de décisions prises par des acteurs mal voyants et dont les options sont à la fois guidées par les héritages qu'ils ont reçus, les contraintes qu'ils subissent, les images du futur qu'ils élaborent et les horizons d'attente qu'ils bricolent. Leur choix n'est pas conditionné par le futur, mais il l'est par l'idée que les décideurs s'en font. Dans le domaine social, héritages et contraintes ont la particularité de peser aussi sur les décisions à travers l'influence qu'ils exercent sur les imaginaires individuels et collectifs.

Pour être applicable, la recherche sur le monde social doit prendre en compte cette dimension. C'est l'objet de la praxéologie. Le développement de celle-ci conduit à la formation de doublets : aux disciplines universitaires classiques se juxtaposent des domaines appliqués qui prennent en compte les dimensions propres à l'action humaine. Cette évolution est en cours en géographie. On le voit dans le domaine politique : la géographie politique y est complétée par la géopolitique (Claval, 2010). Pour expliquer le monde, celle-ci recense les acteurs, analyse l'information dont ils disposent et explore les représentations qu'ils ont reçues de leur milieu ou élaborées par eux-mêmes. La géographie des transports et de la communication est de la même façon doublée par une discipline nouvelle : la logistique.

Dans la mesure où l'enseignement supérieur se doit de former les étudiants à l'action, la place qu'il fait à la praxéologie va croissant. Le succès des formations aux affaires, au tourisme, à la logistique, vient de là.

Dans la mesure où les décideurs poursuivent des objectifs qui mêlent le social, l'économique, le culturel et le politique, et se soucient de leur traduction spatiale, la perspective praxéologique ignore les frontières entre les disciplines classiques. Elle emprunte aux unes et aux autres. L'organisation de l'espace résulte de choix dans lesquels les intéressés ont des objectifs complexes. C'est pour cela que les mixtes, les mélanges, les hybrides sont à la mode dans la recherche actuelle : celle-ci analyse des réalités où plusieurs logiques sont simultanément à l'œuvre, alors que les disciplines classiques se consacraient à une logique spécifique.

Par leur réflexion sur le rôle des autres mondes, des au-delàs ou des en-deçàs dans le fonctionnement des sociétés, la géographie contribue de manière originale au tournant praxéologique des sciences sociales.

Les problèmes actuels de la discipline sont tout autant liés au tournant praxéologique des sciences sociales qu'au tournant culturel qui lui est propre : quelle place revient à la géographie dans les domaines praxéologiques que constituent l'urbanisme, l'aménagement du territoire, les études du transport et de la logistique, la géopolitique, etc ? Comment doit-elle se combiner à d'autres enseignements?

\section{5- Les nouvelles conceptions des humanités}

La culture occidentale repose dès l'Antiquité et de nouveau à partir de la Renaissance sur l'étude des humanités. On s'initie à celles-ci en lisant et en commentant les grands textes littéraires, en assistant à des représentations théâtrales, à des opéras et à des concerts, en admirant les plus beaux tableaux, les plus belles 
sculptures, les plus beaux bâtiments. Au stade suivant, on apprend à écrire, à imaginer des pièces, à faire de la musique, à peindre, à sculpter, à composer. Grâce à l'accès aux lettres et aux arts, la première phase élargit la compréhension que l'on a du monde et des hommes. La seconde assure la maîtrise de modes d'expression littéraires et artistiques qui rendent les hommes plus profondément humains : en apprenant à analyser et à exprimer ce qu'ils ressentent, ils développent aussi leur aptitude à exercer une influence sur les autres.

Dans l'Antiquité grecque et romaine, les humanités demeuraient proches de l'initiation aux mythes à laquelle elles s'étaient substituées. Il s'agissait d'activités laïques, mais qui parlant de la vie et de ceux qui la perdent, de la passion et de la liberté, de la beauté, de l'harmonie ou du sublime, exploraient la condition humaine jusqu'à ses limites, celles où l'existence côtoie la mort et invite à la réflexion religieuse. Telles que les érudits de la Renaissance les font revivre, les humanités nous ramènent à ce que l'Antiquité proposait de meilleur ; elles nous font redécouvrir des formes de sagesse et des philosophies que le christianisme avait fait oublier et nous ouvrent aux lettres et aux arts. Dans l'ambiance néo-platonicienne qui les caractérise d'abord, elles nous initient à l'harmonie des formes conçues selon les canons de la beauté. Il n'y a pas d'humanités sans érudition, mais pour être pleinement efficace, la formation aux humanités demande aussi une expérience directe du monde dans lesquels les Anciens vivaient et où sont apparus leurs successeurs modernes : pour les aristocrates anglais, l'initiation aux humanités se terminait par le Grand Tour, qui leur faisait découvrir l'Italie et plus tard la Grèce et l'Orient.

Ainsi comprises, les humanités s'opposent aux sciences par la place qu'elles accordent à la tradition, par l'usage qu'elles réservent à l'écriture, au dessin, à la peinture, à la musique et à tous les arts et par leurs dimensions philosophiques. Elles parlent des qualités qui rendent les hommes vraiment humains plus que du volume de ce qu'ils produisent. Francis Bacon conseille au chercheur de se défier 'des mots de la tribu' : le divorce entre sciences et humanités s'accomplit ainsi.

La situation change parce que les humanités évoluent. Dans le courant du XIXe siècle, elles se chargent d'histoire. On ne se contente plus de donner en exemple aux adolescents les meilleurs textes des meilleurs auteurs : on leur enseigne les genres littéraires et leur évolution. L'initiation au français se fait désormais sous l'égide de Gustave Lanson et de l'histoire de la littérature. L'histoire de l'art se développe parallèlement.

La recherche que l'on mène dans le domaine des humanités ressemble de plus en plus à celle que pratique l'histoire - l'histoire des idées en particulier. N'est-ce pas grâce à des littéraires comme Pierre Mesnard que l'on sait comment la pensée politique se forme au XVIe siècle dans l'ambiance du conflit entre catholiques et protestants? N'est-ce pas Daniel Mornet qui fait découvrir l'émergence du sentiment de la nature au
XVIIIe siècle ?

Nouvelle étape dans l'évolution des humanités : à la phase historienne, qui court de la fin du XIXe siècle aux années 1930, succède une phase où la linguistique donne une orientation structuraliste au travaux des humanistes. Ceux-ci se présentent désormais comme des spécialistes du discours. Comment ne pas faire appel à eux au moment où l'on découvre que beaucoup de travaux de sciences sociales ne sont que de grands récits?

Dans le même temps, les sciences de l'homme et de la société connaissent un tournant culturel : leur ambition n'est plus seulement de rendre compte, en termes de causalité, des situations qu'elles analysent ; elle est de comprendre les représentations que celles-ci suscitent et leurs effets sur la dynamique des groupes observés. La place accordée aux développements narratifs est de plus en plus grande : la barrière qui existait entre humanités et sciences de l'homme et de la société disparaît.

Rien ne le montre mieux que certains ouvrages. Un exemple : dans celui dirigé par Iain Robertson et Penny Richards, Studying Cultural Landscapes (2003), la moitié des contributeurs enseigne l'anglais, la littérature anglaise, les études juives ou travaille dans des Instituts d'Humanités.

\section{6- Des épistémologies de la curiosité aux épistémo- logies du désir}

\section{1- La connaissance, la curiosité et le désir}

Depuis la fin du XIX ${ }^{e}$ siècle, les géographes soulignent le rôle du terrain dans leur discipline, mais ils ne s'attardent guère sur la manière dont il est mis en œuvre. Il faut attendre les années 1970 ou 1980 pour que des études s'y intéressent : on trouve désormais réductrices les approches exclusivement intellectuelles de la science; on prend désormais en considération les 'pratiques' sur laquelle elle s'appuie (Robic, 2000 ; Baudelle et al., 2001).

Cela traduit une évolution de la conception du savoir, qui conduit à une réflexion plus poussée sur les fondements de l'épistémologie. On cesse de privilégier le 'mouvement des idées' : l'aspect concret des démarches et leurs conditions matérielles sont désormais pris en compte.

Le mouvement s'accentue dans les années 1990. Cela est symptomatique de la mutation de l'image de la science et de la nature de l'épistémologie qui intervient alors. Ce qui motivait la pensée scientifique, c'était la curiosité. Le terme, apparu en français à la fin du XII ${ }^{\mathrm{e}}$ siècle, désignait d'abord, 'le soin, le souci qu'on a de quelque chose'. Il s'applique par la suite à la 'tendance qui porte à apprendre, à connaître des choses nouvelles' (Robert). C'est un 'appétit', une 'soif de connaître'. Dans cette perspective, la science résulte d'un mouvement de l'esprit, qui le porte à explorer le réel pour le 
comprendre et l'expliquer.

La géographie naît ainsi de la tendance à connaître les milieux proches et ceux qui s'étendent au-delà de l'horizon et que l'on découvre en voyageant. A la fin du Moyen Age et à la Renaissance, lorsque l'épistémologie moderne commence à se constituer, elle se nourrit du regard que l'on promène sur un monde que l'on découvre parce qu'il est inondé de lumière. Depuis Roger Bacon et Robert Grosseteste, au XIII ${ }^{\mathrm{e}}$ siècle, un courant de la pensée chrétienne s'intéresse à la lumière parce qu'elle a été créée par le Seigneur dès le premier jour de la Genèse : elle apparaît comme l'énergie que Dieu met en œuvre pour modeler l'univers - comme le véhicule de sa Grâce.

$\mathrm{Au} \mathrm{XV}^{\mathrm{e}}$ siècle, la réflexion des Platoniciens de Florence va dans le même sens comme le souligne André Chastel :

"Les principes philosophiques de la perspective se ramènent en effet à l'idée que l'espace est entièrement traversé par la lumière (il est par là intelligible') et de structure mathématique ('il est par là mesurable'). Ces deux points, que l'on trouvait déjà chez certains savants du XIII ${ }^{\mathrm{e}}$ siècle, occupent une place centrale dans la 'physique' du XV siècle et dans la doctrine de Ficin. Un de ses traités de jeunesse, Quaestiones de luce, insiste sur le fait que la propagation des rayons n'est pas un déplacement d'éléments corporels. La lumière est cosa spirituale et ne peut engendrer que des effets intelligibles. Le commentaire au Timée consolidera cette intuition par la théorie de l'âme du monde et la conception mathématique de l'espace qui en découle... Tel est l'ordre platonicien qui développe l'intuition du cosmos harmonieux" (Chastel, 1982, p. 305306).

Cette conception du savoir se modifie sous l'influence d'une autre composante de la pensée médiévale, le nominalisme, qui impose, pour cerner la vérité, la garantie de l'expérience personnelle des choses. Le dialogue entre l'esprit de l'individu et le monde s'effectue grâce au jeu du regard et de la lumière.

Le corps n'est impliqué dans la construction de la vérité qu'à travers la vue - qui échappe, puisqu'elle perçoit une chose spirituelle, aux déterminations matérielles. Cela a plusieurs conséquences. 1- La cartographie tient une place essentielle dans la géographie car elle résume et met à la portée de tous ce que l'œil du voyageur découvre. 2- La géographie explore le monde à travers la vue, ce que renforce, à partir de Humboldt, au début du XIX ${ }^{\mathrm{e}}$ siècle, l'accent mis sur l'expérience directe de l'espace étudié et sur les paysages que le chercheur y découvre et que l'image permet de communiquer. 3- Science du regard, la géographie apparaît évidemment comme un outil de surveillance, comme le soulignent, depuis une quarantaine d'années, les travaux inspirés par le Surveiller et punir de Foucault.

\section{2- Les épistémologies critiques du terrain}

La transformation des perspectives épistémologiques résulte en bonne partie d'une façon nouvelle d'analyser la démarche des chercheurs - en général et celle des géographes - en particulier. Isabelle Lefort (2012, p. 472-475) parle du 'désir de terrain' :

"Ce qui meut le géographe au terrain, ce n'est pas seulement le désir d'entrer dans la carrière, c'est le désir tout court de s'approprier un pan du monde et qu'on lui en soit reconnaissant [...]. Ainsi vécu et exprimé, le rapport au terrain n'engage sûrement pas le seul intérêt intellectuel du géographe, mais toute sa personne, dans ses dimensions psychologiques et intimement personnelles" (Lefort, 2012, p. 472).

Dans cette nouvelle perspective, le géographe est motivé par le désir plus que par la simple curiosité. Toute la discipline s'en trouve modifiée : elle n'est plus le fait de purs esprits. Elle a des assises corporelles, trop longtemps négligées. Il faut réparer cet oubli. C'est ce à quoi des collègues commencent à s'employer, dans le monde anglo-saxon, dans les années 1990 :

"Dès le début des années 1990, le problème du terrain surgit dans la géographie anglophone sous la double impulsion du développement d'une épistémologie féministe [...] et du développement des qualitative studies - au déploiement et à l'encodage desquelles les géographes féministes participent, à côté de la tradition phénoménologique et de l'ensemble des courants poststructuralistes" (Volvey et al., 2012, p. 446).

Que le courant des qualitative studies soit impliqué dans cette réévaluation du rôle du terrain dans la géographie, rien de plus normal : depuis Humboldt, c'est de l'observation visuelle directe que naît l'apport le plus précieux à la géographie. Le rôle des géographies du genre vient, en revanche, de la place nouvelle accordée à la corporéité du chercheur. Ce qui est en cause, " c'est le 'régime scopique' de connaissance de la géographie classique (élaboration de données produites dans l'observation visuelle) ». On voudrait le remplacer par " un 'régime haptique' de connaissance (un régime basé sur l'élaboration scientifique de données pré-linguistiques, soit haptiques ou empathiques) [...] » (Volvey et al., p. 453-454).

Une interprétation 'genrée' de la géographie s'esquisse ainsi. La pratique masculine du terrain, « calquée sur celle de l'exploration, évolue entre possession par l'arpentage, pénétration par le regard et contrôle par le recouvrement exhaustif d'un espace extérieur [...] féminisé [...]» (Volvey et al., p. 447). La motivation inavouée de cette forme de pratique ? "La stratégie de confirmation ou consolidation de l'identité sociale, 
masculine, du chercheur » (Volvey et al., p. 447).

Une autre pratique du terrain est concevable : elle serait dominée par le souci de l'autre - le care, pour employer le terme anglais - qui caractérise les attitudes féminines.

"L'enquête de terrain féministe [...], dotée d'une échelle d'opération réduite (micro-procédures) et fondée sur l'interlocution a été placée au fondement d'un feminist political project within the discipline. [C'est] un projet d'empowerment réciproque du sujet cherchant et du (ou des) sujet(s) enquêté(s) qui font entendre et représentent leurs voix $[. .$.$] jusqu'à participer d'une stratégie$ d'activisme politique [...]" (Volvey et al., p. 447).

Voilà le programme d'une épistémologie qui considère que la connaissance scientifique a jusqu'ici été gâtée par la suprématie masculine ; il convient d'imaginer de manière plus 'pure' le travail en extérieur.

\section{3- Le développement des épistémologies critiques de la géographie en France}

Le développement des épistémologies critiques est plus récent en France que dans les pays anglophones. Il y prend différentes formes. Pour Yann Calbérac, " le terrain des sciences sociales apparaît comme le pendant du laboratoire des sciences expérimentales » : c'est en analysant sa pratique que l'on rompra enfin avec les épistémologies traditionnelles :

"Faire une histoire de la géographie sous l'angle du terrain invite donc à dépasser les représentations que les géographes se font de leur discipline pour interroger les discours que le terrain ne cesse d'alimenter" (Calbérac, in Volvey et al., p. 452).

C'est à cette question qu'il consacre sa thèse (Calbérac, 2010).

Anne Volvey choisit un angle différent :

"Elle s'est intéressée à la pratique du terrain des géographes. Elle interroge alors sa dimension subjective, qu'elle met au centre de sa réflexion pour en faire un enjeu en deçà du cognitif, et sa dimension spatiale, qu'elle instaure en moyen de cet enjeu" (Volvey et al., p. 452).

Son propos la conduit à souligner ce qui bloque le développement des épistémologies haptiques à la mode des géographes anglophones :

"Si les géographies anglophones contemporaines ont bien substitué un 'régime linguistique' (élaboration de données produites dans l'interdiscursivité) au 'régime scopique' de connaissance de la géographie classique (élaboration de données produites dans l'observation visuelle), elles peinent en revanche à fonder un 'régime haptique' de connaissance (un régime basé sur l'élaboration de données pré-linguistiques, soit haptiques ou empathiques) [...]. De fait, elles n'adoptent pas le principe de symétrie qui leur permettrait d'envisager pleinement un régime haptique de connaissance à partir d'une réflexion critique fondée sur le rapport entre la méthodologie de terrain fondée sur le modèle du care, la dimension spatiale de celle-ci et l'expérience subjective du chercheur (Volvey et al., p. 453-454)."

Qu'apportent ces épistémologies critiques?

\section{4- Les épistémologies du désir face aux épistémologies de la curiosité}

L'intérêt nouveau pour le terrain résulte de la mutation que subit, depuis une trentaine d'années, la conception même que l'on se fait de l'épistémologie : la connaissance cesse de répondre à l'éveil de curiosités, c'est-à-dire à un besoin fondamentalement intellectuel. Elle répond à une motivation plus profonde et plus générale : le désir.

Cette mutation s'inscrit dans un mouvement plus ample : le chercheur n'est jamais isolé dans sa tour d'ivoire ; ses réflexions portent la marque de sa constitution physique, de la formation qu'il a reçue, du milieu social et intellectuel ou il évolue et des politiques qui y sont menées. Le savoir qu'il élabore doit être remis dans son contexte : la pensée ne se meut pas dans un environnement sans frictions et sans pesanteurs. La connaissance qui résulte alors du traitement des données est 'située' - c'est-à-dire non universelle.

Les épistémologies critiques insistent depuis longtemps sur le fait que le travail scientifique reflète toujours la 'positionnalité' de celui qui le mène : c'était déjà le cas de l'historicisme ; c'est le cas de beaucoup de sociologismes ou d'économismes, de celui, par exemple d'un certain marxisme, qui voulait que l'économique (l'état des forces productives et les modes de production) décide toujours de tout en dernière instance. Les épistémologies de la corporéité n'échappent pas à la critique que toutes ces conceptions appellent: «Qu'est-ce qui garantit la validité du savoir scientifique dans l'épistémologie féministe et les méthodes qualitatives en général ? » (Volvey et al.,, p. 449).

En remplaçant un savoir 'situé' par son contraire, on ne bâtit pas un savoir universel. Les collègues francophones qui explorent ces nouvelles pistes de recherche sont conscients de la fragilité de ce type de savoirs. Pour s'en affranchir, Yann Calbérac s'inspire de Bruno Latour et de sa théorie de l'acteur-réseau. Anne Volvey tire parti de variantes de la psychanalyse qui rendent compte du rôle de la subjectivité dans les pratiques de terrain.

Il est évidemment nécessaire de mesurer ce qui pèse sur le chercheur, oriente ses travaux, le séduit ou lui répugne lorsqu'il est confronté au monde. La conquête du savoir est faite de batailles renouvelées. Elle ne comporte pas de victoire qui ouvrirait définitivement à l'esprit humain le continent du savoir : l'univers 
platonicien des Idées n'existe pas ; la connaissance se construit; ses résultats ne sont jamais que provisoires.

Certains tirent de ce constat la conviction que les formes traditionnelles de la connaissance sont sans fondement. Il faut, certes, les soumettre à révision, critiquer leurs résultats, rabattre leurs ambitions. La prise en compte du terrain et de la dimension corporelle qu'il revêt ne remet cependant pas en cause l'idée que l'évolution de la géographie reflète un certain nombre de logiques liées à l'histoire des idées, à la dynamique des institutions, à l'ambition des chercheurs et à la demande sociale. On ne gagnerait rien à oublier ces déterminations pour ne tenir compte que de celles que prennent en compte les épistémologies du désir.

\section{7- La remise en cause de l'idée de société}

Les conceptions que l'on se fait de la société changent : la plupart des groupements humains ne sont ni des communautés au sens de Tönnies (c'est-à-dire des groupes à solidarité mécanique, au sens de Durkheim), ni des sociétés à solidarité organique (au sens de Durkheim). Elles sont marquées par la présence de nombreux marginaux et par l'exclusion : les sociétés ne sont donc pas forcément des constructions solidaires ; le lien social n'est pas universel. Des problèmes que l'on avait jusque-là ignorés surgissent alors : celui de l'identité en tout premier lieu (celle-ci ne va de soi que dans les groupements à lien social sur lesquelles l'attention s'était jusqu'alors focalisée) ; celui aussi de l'existence de contre-sociétés, de contre-cultures, d'antimondes, selon l'expression de Roger Brunet, ou d'hétérotopies, selon celle de Michel Foucault.

\section{1- Société et solidarité territoriale}

Au XIX ${ }^{e}$ siècle, la pensée sociologique se développe dans un cadre simple. La société existe avant l'individu, qui ne pourrait survivre sans elle. La réalité sociale combine tradition et évolution. Elle permet à des personnes dont le statut, les responsabilités et les occupations sont diverses de coopérer aux tâches nécessaires à la survie de tous. Tout groupe doit assurer la reproduction biologique de ses effectifs, la transmission de sa culture, la production des biens matériels et symboliques dont ses membres ont besoin et la résolution des tensions et conflits inhérents à la diversité des comportements et des situations. Dans cette régulation, la religion et l'idéologie tiennent un rôle fondamental.

L'idée de société devient ainsi une réflexion sur le rôle du lien social, cet ensemble d'obligations, de sentiments et de valeurs qui assurent la vie et la cohérence des groupes.

L'idée que dans un cadre territorial donné, tous les hommes partagent quelque chose était absente du monde antique ; dans la cité grecque, la solidarité n'existait qu'entre citoyens ; elle ne s'étendait ni aux esclaves, ni aux métèques (les étrangers qui vivaient dans la cité). Le sentiment qu'un lien unit toutes les personnes qui résident en un même lieu, dans une même région ou dans une même nation apparaît progressivement dans la chrétienté médiévale. Elle se précise durant la Renaissance.

La pensée sociale qui émerge au XIX ${ }^{\mathrm{e}}$ siècle reprend ce thème et insiste sur la solidarité inhérente à la vie des groupes. En France, Pierre Leroux incarne ce nouveau courant au début des années 1830. Le sociologue Léon Bourgeois et l'économiste Charles Gide le systématisent à la fin du XIX ${ }^{\mathrm{e}}$ siècle.

L'inspiration solidariste de la pensée sociale, particulièrement forte en France, s'impose dans toutes les sociétés occidentales dans la première moitié du XX siècle : elle motive les politiques de bien-être social qui apparaissent dès 1900 et s'affirment dans les années 1930, durant la Seconde Guerre mondiale et immédiatement après.

\section{2- Modernisation et société nationale}

Les sociétés changent beaucoup au XIX ${ }^{\mathrm{e}}$ siècle. Les sociétés d'Ancien Régime étaient essentiellement rurales. La vie économique demeurait locale. Avec la révolution industrielle, la situation se transforme. La migration de beaucoup de journaliers et de petits paysans des zones rurales vers les nouveaux foyers industriels et les grandes villes fait naître la société moderne : il y a changement d'échelle et de fonctionnement.

Dès le début du XIX ${ }^{e}$ siècle, la curiosité des spécialistes de la vie sociale est attirée par les nouvelles caractéristiques de la vie collective ; cela peut se vérifier dans l'œuvre de Saint-Simon. Mais la réflexion sur la modernisation se précise surtout avec Ferdinand Tönnies (Tönnies, 1887).

"L'idée de société ne peut être dissociée d'une définition de la modernité. L'œuvre de Tönnies, Communauté et société [...] est la matrice d'un discours de la modernité dans lequel la société est, en premier lieu, moderne" (Dubet, 2006, p. 1085).

Tönnies pose clairement le problème. Comme le dit Dubet :

"La société est moderne parce qu'elle est dominée par la division du travail, par la complexité croissante des statuts, des institutions et des organisations. La question sociologique centrale s'impose dès lors : comment expliquer l'ordre social en dépit de la différenciation continue des pratiques sociales, des inégalités qu'elle génère et de l'anomie qu'elle provoque" (Dubet, 2006, p. 1085).

Pour Tönnies, le passage de la communauté à la société provoque un appauvrissement du lien social : dans les petits groupes du monde rural traditionnel, tout le monde se connaissait. Quand une famille rencontrait des difficultés, les autres l'aidaient. Dans une société, les relations sont de courtoisie, mais anonymes. Le vendeur est attentif, mais il ignore les problè- 
mes de ceux qui lui achètent et ne cherche pas à les connaître. Beaucoup de gens vivent à côté de zones de misère, mais ne font rien pour secourir les personnes qui y sont en difficulté.

La majorité des sociologues utilise les catégories introduites par Tönnies, mais selon une perspective moins pessimiste. Emile Durkheim sait que les sociétés modernes sont menacées par leur déficit de générosité, mais il pense qu'une autre forme de solidarité, la solidarité organique, se substitue à la solidarité mécanique qui présidait à la vie des communautés (Durkheim, 1893). Si l'on parvient à y susciter une conscience plus forte des devoirs de chacun, leur cohésion sera maintenue.

Pour d'autres sociologues de l'époque, Max Weber par exemple, la caractéristique essentielle des sociétés modernes est le rôle toujours plus fort qu'y joue la rationalité. De nouveaux types d'institutions se développent : bureaucraties publiques de l'Etat moderne, bureaucraties privées des entreprises. Grâce à leurs interventions, de nouvelles formes de solidarité peuvent être imaginées - celles du bien-être social par exemple. Ainsi conçue,

"La société est non seulement moderne. Très souvent, elle est perçue comme un système dont l'intégration est fonctionnelle avant même d'être subjective et culturelle. La société apparaît dès lors comme un monde organisé et cohérent, dans lequel chaque mécanisme culturel et social a la propriété de maintenir l'ordre social [...].

"Ainsi conçue, la notion de société peut être considérée comme une construction analytique, comme un ensemble de concepts plus ou moins organisés grâce auxquels les sociologues essaient de combiner la question de l'ordre social avec celle de son évolution" (Dubet, p. 1086).

La notion de société ainsi élaborée apparaît d'autant plus intéressante qu'elle est proche des modèles économiques et met l'accent sur les activités productives et la satisfaction des besoins matériels. Elle se prête à l'élaboration d'approches théoriques. Un de ses caractères les plus intéressants est sa dimension spatiale, celle de l'Etat-nation :

"La sociologie a construit des discours dans lesquels la société est devenue le personnage central. Mais le succès de ces discours ne venait pas de leur valeur intellectuelle [...]. Elle résultait aussi de ce que la notion de société se rapportait aussi aux Etats-nations" (Dubet, 1086).

\section{3- La crise de la notion de société}

La globalisation a commencé avec les Grandes Découvertes, mais jusqu'au milieu du XIX ${ }^{\mathrm{e}}$ siècle, la portée de la plupart des flux de biens, de personnes et d'informations demeurait limitée : l'essentiel de la vie des groupes humains était contenu dans des espace finis et dont les frontières étaient nettes. Il était donc possible de parler de sociétés, c'est-à-dire d'entités sociales encloses dans un territoire dont elles assuraient l'exploitation et l'organisation.

Dans le monde traditionnel, ces unités sociales étaient petites - quelques centaines ou quelques milliers de personnes vivant dans des aires de quelques dizaines ou quelques centaines de kilomètres carrés. La situation change avec la construction de l'Etat moderne. En Occident, celle-ci débute avant la Renaissance, mais la transition entre l'Etat monarchique et l'Etat national n'intervient qu'à la fin du XVIII ${ }^{\mathrm{e}}$ siècle ou dans le courant du XIX ${ }^{\mathrm{e}}$. La majorité des relations sociales s'inscrit dès lors à l'intérieur des frontières qui réunissent un même peuple. La réalité que les sociologues et les autres spécialistes des sciences sociales étudient est faite de sociétés nationales. Une forte solidarité entre leurs membres paraît naturelle. Les chercheurs n'éprouvent pas le besoin de s'interroger sur quelque chose qui va de soi :

"Comme beaucoup d'animaux, les hommes vivent en société : et pourtant, pas plus que les ouvrages de biologie n'incluent d'article sur 'la vie', la majorité des encyclopédies et des dictionnaires de sciences sociales n'incluaient d'article sur 'la société" (Dubet, 2006, p. 1084).

La mobilité croissante des cinquante dernières années modifie la situation (Dubet et Martuccelli, 1998 ; Martuccelli, 1999). Les frontières cessent d'apparaître clairement dessinées : les faisceaux de relations économiques, sociales et culturelles dans lesquelles chacun s'intègre les chevauchent désormais. Les gens sont prêts à manifester leur solidarité pour les victimes d'une famine ou d'un tremblement de terre à l'autre bout du monde, mais n'éprouvent aucune compassion pour les pauvres qui vivent à côté d'eux. Peut-on parler d'une société ? A coup sûr, non!

\section{4- La crise des identités}

Chacun a une identité - ou une série d'identités hiérarchisées et emboîtées - mais jusqu'il y a cinquante ans, les spécialistes de sciences sociales ne parlaient pas plus d'identité que de société. Chacun appartenait à un groupe et en avait conscience ; le développement des sentiments d'identité résultait de l'expérience vécue par chacun. Les problèmes d'identité ne retenaient l'attention que dans deux circonstances : (i) en cas de conversion d'une religion à une autre, du catholicisme au protestantisme ou du christianisme à l'islam ; (ii) lors de la défaite d'un peuple et de la destruction de sa culture par les vainqueurs, comme dans le cas des Aztèques face à Fernando Cortez ou des Incas face à Francesco Pizzaro.

La situation est aujourd'hui différente (Zelinsky, 2001) : beaucoup d'individus ne savent plus qui ils sont. En France, un certain nombre d'enfants des immigrants maghrébins n'ont pas le sentiment d'être fran- 
çais lorsqu'ils sont en France, et celui d'être tunisiens, algériens ou marocains quand ils sont en Afrique du Nord.

Pour beaucoup de Français modestes qui habitent à la périphérie des grandes villes, le doute existe aussi. A l'heure de l'Europe unie, le fait de vivre en France ne leur assure plus un avantage considérable : leur situation serait à peu près la même s'ils vivaient en Allemagne, en Angleterre, en Espagne ou en Italie. L'urbanisation leur a fait perdre la forte identité locale ou régionale qui consolidait les identités nationales du passé : ils n'ont plus les mêmes raisons que leurs parents de se sentir français.

La crise des identités est un problème général des sociétés contemporaines ; il résulte en grande partie de la mobilité accrue et des migrations nationales et internationales.

\section{5- De la notion de classe sociale à celle d'exclusion}

Les sociétés ne sont jamais homogènes. Pour rendre compte des différences de statut, de richesse, de revenu et de travail, les historiens français du début du $\mathrm{XIX}^{\mathrm{e}}$ siècle inventent la notion de classe sociale. Marx et les sociologues lui confèrent un rôle central dans l'interprétation qu'ils proposent des sociétés en voie de modernisation. Jusqu'au milieu du XX $\mathrm{X}^{\mathrm{e}}$ siècle, l'existence de différences importantes au sein des collectivités nationales y suscite des conflits, mais n'empêche pas leur fonctionnement : la dynamique sociale résulte de ces tensions et des efforts consentis pour les surmonter.

La situation actuelle est différente. La lutte des classes n'a pas disparu, mais le rôle qu'elle tient est certainement plus faible qu'il y a un siècle. A la fin des années 1960, Henri Lefebvre soulignait déjà la diversité croissante des mouvements sociaux ; leurs revendications portaient sur le temps libre ou sur un meilleur accès à la culture - ou à la ville - autant que sur l'augmentation des salaires (Lefebvre, 1968).

Il faut donc mettre en œuvre d'autres catégories pour appréhender cette réalité nouvelle. Florence Hagel en esquisse les contours :

"La classification en termes d'exclu se substitue effectivement de plus en plus fréquemment à la perception en termes de catégories sociales, forme fortement instituée et très longtemps dominante de classification de la société. Parler en termes d'exclusion implique l'adhésion à la représentation d'un monde coupé en deux et donc l'acceptation d'une vision de la société comme réalité construite sur une opposition entre un extérieur et un intérieur. Cette catégorisation s'inscrit [...] dans un projet normatif, puisque cette coupure est condamnée sans qu'un responsable soit désigné : l'état d'exclu renvoie généralement à des mécanismes invisibles, globaux et complexes" (Hagel, 2003, p. 493).
Les problèmes de pauvreté et de misère n'ont pas disparu, mais ce qui paraît nouveau et grave, c'est la présence de groupes rejetés par tous; depuis cinquante ans, les problèmes de l'exclusion ne cessent de s'aggraver ; les études qui leur sont consacrées se multiplient (Paugam, 1996 ; Sibley, 1995 ; Varii Auctores, 1996). Nous vivons dans des systèmes où la solidarité ne tient plus un rôle central. Cette évolution va de pair avec la globalisation.

\section{6- Le recul de l'idée de société}

Jusqu'au milieu du XX' ${ }^{\mathrm{e}}$, les sciences sociales continuent à utiliser les conceptions de la société élaborées au XIX ${ }^{\mathrm{e}}$ siècle. La perspective fonctionnaliste est alors symbolisée par Talcott Parsons (1951). La situation est aujourd'hui différente :

"La représentation de la société comme un organisme fonctionnel ne cesse de diminuer dans la production sociologique des trente dernières années : les systèmes sociaux sont moins conçus comme des ensembles donnés avec des frontières nettes que comme les effets émergents des comportements et des choix plus ou moins rationnels des acteurs collectifs ou individuels" (Dubet, 2006, p. 1086).

La sociologie d'Anthony Giddens offre un exemple démonstratif de cette évolution (Giddens, 1984). Pour lui, la société n'existe pas comme une réalité préformée et qui s'imposerait à tous. Elle naît sur des scènes locales (les locales) et résulte de la coalescence de formes locales ou régionales de sociabilité. Comme Benedict Anderson l'a montré, les sociétés nationales ne prennent la forme que nous leur connaissons aujourd'hui qu'au début du XIX ${ }^{\mathrm{e}}$ siècle (Anderson, 1983). Elles résultent alors d'une longue action idéologique et des politiques d'enseignement public. Le cadre national n'a jamais été une réalité première dans le domaine social. Il a d'abord été imaginé, puis construit.

L'évolution des sciences sociales va plus loin : “... la majorité des sociologues contemporains observe une séparation progressive de l'acteur et du système, [...] de la subjectivité des individus et du système, de l'intégration sociale et de l'intégration systémique. Les théories de l'action sociale inspirées, entre autres, par l'individualisme méthodologique, par l'interactionnisme symbolique ou par l'ethnométhodologie, rompent avec une conception de l'action conçue comme un produit de l'intériorisation de modèles et de rôles sociaux. La chaîne des causalités paraît avoir été renversée et [...] l'idée de la société n'est plus indispensable à la pensée sociologique comme elle l'a été pendant longtemps" (Dubet, 2006, p. 1096).

Beaucoup de spécialistes des sciences sociales ne sont pas encore conscients de cette évolution : pour eux, l'idée que la vie sociale s'inscrit normalement dans 
une aire dont les frontières sont nettes - qu'il s'agisse de celles de la communauté locale du monde traditionnel ou de celles de l'Etat national du monde moderne - avait une origine double : (i) elle décrivait des réalités que l'on pouvait observer à la fin du XIX ${ }^{e}$ siècle et au commencement du XX⿳亠丷厂 ; (ii) elle répondait à une question qui paraissait essentielle à l'époque : quelle était l'origine de l'ordre social ? Après la Révolution française et pour plus d'un siècle et demi, le maintien de celui-ci se situe au cœur des préoccupations du monde intellectuel. Cette période est révolue. Il n'existe plus aujourd'hui de cadres territoriaux aux limites nettes et qui enserreraient les groupes. L'idée de société ne s'impose plus avec la même urgence. La vie sociale et économique s'inscrit dans des aires de dimensions variées ; les structures politiques cherchent à s'adapter à cette situation.

La structure sociale du monde ne se caractérise pas par une mosaïque de sociétés nationales : elle apparaît tous les jours davantage comme un mélange de groupes plus ou moins superposés et liés. La société n'est jamais une réalité objective : elle résulte toujours d'une prise de conscience ; sa base est symbolique.

Dans cette perspective, l'intégration de groupes humains dans une entité plus large suppose l'acceptation par ceux-ci d'un même cadre de référence symbolique : une telle situation se rencontrait fréquemment $\mathrm{au} \mathrm{XIX}^{\mathrm{e}}$ siècle et au commencement du XXe, quand la majorité de ceux qui s'installaient dans les agglomérations urbaines dont la croissance s'accélérait venaient de zones rurales et n'avaient pas accès aux formes supérieures de culture : ils acceptaient alors celles que leur offraient leurs régions d'accueil. Aujourd'hui, beaucoup des nouveaux arrivants participent déjà à des formes supérieures de culture ; ils ne sont plus prêts à accepter l'ensemble des valeurs du milieu où ils viennent s'installer.

La leçon que l'on peut tirer de ces observations est simple : l'intégration des individus et des groupes au sein d'une société n'a jamais été automatique ; elle a toujours résulté d'une volonté politique et de l'élaboration de cadres symboliques capables de surmonter la diversité des traditions en fixant des objectifs à partager dans le futur.

Le sinologue François Jullien souligne que jusqu'au milieu du $\mathrm{XX}^{\mathrm{e}}$ siècle, l'intégration d'autres groupes dans les sociétés occidentales était liée à l'universalisme de celles-ci. La pensée occidentale est entrée en crise dans la seconde moitié du $\mathrm{XX}^{\mathrm{e}}$ siècle : son universalisme était fondé sur la puissance de son rationalisme scientifique ; aujourd'hui, cette idée est de plus en plus critiquée ; cela veut-il dire que la situation est sans issue?

"Si nous ne nous fions plus au caractère inné de notions qui seraient données de manière anticipée et si nous nous libérons de toute définition de 'l'Homme', mais sans abandonner pour cette raison le commun de l'intelligible, voici qu'une nouvelle perspective s'ouvre à la pensée" (Jullien, 2008, p. 259).

L'unique solution pour surmonter les différences entre les cultures est de les considérer en ce quelles ont de commun - une manière de concevoir et de construire l'homme :

"Si, au lieu de valoir comme un test idéologique pesant, [l'universel] sert effectivement d'idée régulatrice qui guide la recherche : en ouvrant toute totalité donnée, il, de manière irrépressible, ne cessera pas de libérer à nouveau les conditions de possibilité d'un commun toujours menacé de se réduire et de se retirer : et le sentiment de l'humain ne connaîtra pas de limite [...] pour croître et se développer" (Jullien, 2008, p. 263).

L'idée de société n'a pas l'évidence que lui reconnaissaient il y a un siècle les spécialistes des sciences sociales. Le modèle qu'ils acceptaient décrivait une situation qui prévalait dans les petites cellules du monde rural - les communautés - et qui s'était imposée plus récemment à l'échelle des nations. Le monde contemporain est différemment structuré - comme l'était aussi, on commence à s'en apercevoir, la plupart des sociétés du passé. Il est fait à la fois de sociétés, de contre-sociétés et de contre-cultures, et d'un continuum de situations intermédiaires qu'il importe de préciser.

\section{8- Repenser la société : approches critiques, socio- logie de la justification ou fondation d'une nouvelle sociologie?}

La déconstruction et la remise en cause de concepts de base, comme celui de société, correspondent à une phase de doute et d'incertitude. Celle-ci ne peut durer indéfiniment : la phase suivante est celle de la réforme et de la refondation. Nous les analyserons à partir d'exemples pris chez des auteurs francophones.

\section{1- Les sociologies critiques : l'exemple de Bourdieu}

La première parade au vide créée par la déconstruction, c'est de concevoir des sciences sociales qui ne se contentent plus de décrire et d'expliquer ce qui est, mais qui dénoncent les malfaçons et les injustices de la vie sociale.

L'idéal d'une approche critique est ancien : il naît avec les Lumières ; pour elles, la mise en évidence des injustices dont souffre la société conduira à les éliminer rapidement. Cet espoir est déçu par l'échec de la Révolution française. L'ambition critique de la pensée sociale ne disparaît pas : elle est présente chez Hegel pour qui la course de l'Esprit oriente le devenir social ; la Raison progresse par des voies détournées, si bien que ceux qui la mettent en œuvre n'ont pas conscience de la portée et du sens de ce qu'ils font. Marx fait un travail analogue en dénonçant les dimensions cachées des mécanismes économiques. Les anarchistes, demeurés plus proches des Lumières, cherchent à infléchir le cours de l'histoire en dénonçant le rôle de l'Etat et des 
maux qu'il engendre, l'inégalité, l'oppression, la tyrannie et la guerre.

Les sciences sociales prennent la forme que nous leur connaissons au moment où se développent ces démarches critiques, mais cherchant surtout à rendre compte de ce qui est, elles sont beaucoup plus conservatrices. Entre les deux guerres mondiales et autour de Horkheimer et de Adorno, l'Ecole de Francfort renoue avec l'idée critique. L'exil de ses membres durant l'époque nazie facilite la diffusion de leurs idées dans le monde anglo-saxon. Elles y connaissent un succès foudroyant à la fin des années 1960. Dénoncer les malfaçons de l'univers social devient une ardente obligation morale pour la majorité des chercheurs, que ceux-ci soient sociologues, économistes, anthropologues, politologues, historiens ou géographes. A quel niveau appliquer cette critique?

"Il convient de distinguer la critique épistémologique de la sociologie de la critique sociale de la société, alors même qu'une théorie critique se doit de les combiner en traquant systématiquement les réifications et les aliénations" (Vandenberghe, 2006, p. 182).

$\mathrm{Au}$ niveau supérieur de l'épistémologie, certains philosophes s'interrogent sur ce qui peut conférer une dimension critique au savoir. C'est le cas de Roy Bhaskar, dont l'ouvrage, The Possibility of Naturalism (1978) donne naissance au mouvement du Réalisme critique et justifie, dans le domaine épistémologique, le constructivisme radical. L'idée centrale de Bhaskar est simple : le réel a une profondeur certaine, car il est stratifié : c'est à partir de cette proposition ontologique que toute sa réflexion se développe. Le chercheur est conditionné par l'environnement dans lequel il vit (un thème qui vient de la sociologie allemande de la connaissance), mais il ne l'est pas totalement : il est capable d'aller au-dessous de la surface des choses pour mettre à jour les niveaux cachés du réel - mais sans pouvoir jamais atteindre les plus profonds.

"[Bhaskar distingue] les dimensions 'transitive' (épistémique) et 'intransitive' (ontologique) de la connaissance (Bhaskar, 1978, p. 17). Appliqué à la nature, le principe de non-transitivité existentielle des objets de connaissance pose seulement que la nature existe indépendamment des observations et des descriptions que nous en faisons. [...] Le principe de transitivité historico-sociale de la connaissance sociale des objets reconnaît que la nature ne peut être connue que sous certaines descriptions et que celles-ci sont socialement et historiquement valables" (Vandenberghe, 2006, p. 42).

La nature existe sur le plan ontologique, mais sur le plan épistémologique, elle est construite par les hommes. Une science critique du social est ainsi possible : alors que la société nous paraît aller de soi, comme une chose naturelle, c'est une réalité que les hommes ont édifiée.
Dès The Possibility of Naturalism, la proximité des thèmes de Bhaskar et de ceux du Marx est évidente. Pour avoir parlé, à ce sujet, de 'marxisme rebouilli', la chronique d'épistémologie que je tenais dans Progress in Human Geography est interrompue en 1983 : un certain nombre de collègues anglophones me considèrent dès lors comme un réactionnaire. La suite de l'œuvre de Roy Bhaskar me donné raison, comme le montre la publication en 1993 de Dialectic. The Pulse of Freedom.

Pierre Bourdieu représente parfaitement ce courant de réflexion épistémologique au sein de la sociologie française. Son œuvre s'appuie sur une théorie de la connaissance qu'il a développée entre 1966 et 1972. Elle relie "systématiquement les notions vénérables de 'champ', 'd'habitus' et de 'violence symbolique'" (Vanderberghe, 2006, p. 185). Les individus sont placés dans des champs, qui conditionnent les vues qu'ils ont sur les positions qu'occupent les uns et les autres. L'habitus les conduit à intérioriser les valeurs liées à la position qu'ils occupent eux-mêmes.

"En appliquant consciencieusement la méthode structurale, le sociologue est à même d'objectiver la réalité sociale comme un système de relations entre personnes et de comprendre la position (et les prises de position) de chaque personne à l'intérieur d'une configuration qui la met en relation avec toutes les autres positions (et prises de positions) et leur donne leur sens objectif, permettant d'expliquer ainsi le sens des actions à partir du réseau complet de relations dans et par lesquelles les actions s'accomplissent" (Vandenberghe, 2006, p. 186).

Dès lors, les réactions des individus sont prévisibles car prédéterminées à la fois par le champ et par l'habitus. La théorie lui révélant ces processus, le sociologue critique est à même de comprendre ce qui fait agir les individus - et qui leur échappe :

"C'est la structure des relations constitutives de l'espace du champ qui commande la forme que peuvent revêtir les relations visibles d'interaction et le contenu même de l'expérience que les agents peuvent en avoir" (Bourdieu, 1982, p. 42).

La démarche structurale s'appuie sur la distinction de trois niveaux dans le réel, celui des comportements observables, celui des relations dans lesquelles ils s'insèrent, et celui des structures qui commandent l'ensemble. C'est parce qu'il est capable de déchiffrer ce dernier niveau que le sociologue éclaire le monde et peut dénoncer ses malfaçons. Mais il le fait en réduisant les individus qu'il étudie au rôle de simples pions sur un échiquier auquel ils ne comprennent rien. Beaucoup de chercheurs s'insurgent contre une telle attitude :

"Alors que la théorie critique de Bourdieu s'attaque à la fois à ceux qui hypostasiaient le substantif en substance et s'appuie sur une tradition hégélo-marxiste pour dénoncer les injustices de la domination de classe, Boltanski-Thévenot critiquent la théorie 
critique pour son arrogance épistémologique et normative..." (Vandenberghe, 2006, p.184).

\section{2- La sociologie de la justification, ou sociologie pragma- tique, de Boltanski et Thévenot}

Une deuxième façon de reconstruire la sociologie se développe en France depuis les années 1980. Elle est l'œuvre de Luc Boltanski et de Laurent Thévenot (1991). Elle est axée sur l'analyse de situations de conflit - sur la microsociologie plus que sur la macrosociologie. Elle part de l'analyse très précise de situations concrètes et dresse un tableau détaillé des attitudes, des positions et des décisions de ceux qui en sont les acteurs. Chacun expose et défend la légitimité de sa position et les avantages qui doivent lui revenir. L'issue des conflits ne dépend pas seulement, ou essentiellement, de la force relative des participants. Elle résulte du sens de la justice dont chacun se réclame, et des formes de justification qu'il fait siennes.

La réalité qu'analysent Boltanski et Thévenot (1991) ne présente plus que deux niveaux : celui des situations analysées, et celui des répertoires d'arguments auxquels les protagonistes font appel :

"Pour comprendre les pratiques, il faut désormais faire un détour herméneutique par des conventions métaphysiques que les gens ordinaires invoquent dans des situations de dispute. Ce sont elles qui orientent les pratiques et leur donnent un sens. Refusant d'invoquer des mécanismes sociaux et des forces inconscientes qui déterminent l'acteur, la sociologie pragmatique rompt avec le 'paradigme du dévoilement' des maîtres de la suspicion (Marx, Nietzsche, Freud) pour se rallier au paradigme de l'interprétation des herméneutes et des phénoménologues. Insistant davantage sur ce que l'être humain fait que sur ce qui est fait de lui, la sociologie de la justification appréhende l'être humain comme un être libre [...]. A la différence de la sociologie critique, elle prend les discours, les principes et les valeurs légitimateurs (sic) qui donnent un sens à l'action au sérieux, sans y voir des illusions bien fondées qu'il faudrait soumettre à la critique au nom d'une connaissance supérieure, au nom de la Science. Contrairement au monde tridimensionnel de la domination, le monde bidimensionnel de la justification est une monde sans structures profondes à dévoiler et donc sans illusions à désigner" (Vandenberghe, 2006, p. 195-196).

Dans le vocabulaire mobilisé par la sociologie pragmatique de la justification, les recueils d'arguments justificatifs auxquels font appel les individus qui s'opposent ou s'épaulent dans une situation concrète s'appellent des 'Cités' - allusion faite aux valeurs dont se réclament ceux qui plaident leur cause en tant que citoyens :
"Pour configurer l'action et agir en commun, les acteurs doivent désormais faire un détour par la culture et puiser dans le fonds commun des représentations collectives. Grâce aux Cités, on dépasse les platitudes de l'empirisme de l'ethnométhodologie et de la théorie des acteurs en réseaux pour aboutir à une construction théorique à deux étages (individuel/collectif, particulier/général) où l'on retrouve les entités relevant des trois ontologies régionales, à savoir des personnes humaines (des âmes) en interaction avec des objets et des choses (des corps non animés), ainsi qu'avec des êtres métaphysiques (des esprits) qui médiatisent les interactions entre les personnes et les objets et, ce faisant, permettent de les mettre 'en équivalence' et, donc, de les qualifier" (Vanderberghe, 2006, p. 203).

Ces 'Cités' reposent sur des principes communs : "Conçus comme des ordres axiologiques construits autour de 'principes supérieurs communs' qui servent comme des repères et des répertoires de légitimation, le modèle axiomatique des Cités formule trois contraintes que toute philosophie politique doit prendre en considération : primo, personne ne peut être exclu d'une cité (contrainte de commune humanité) ; secundo, chacun peut accéder à toutes les cités (contrainte cosmopolitique) ; tertio, chaque membre d'une cité peut être ordonné selon un principe de grandeur et être qualifié de 'grand' ou de 'petit' (contrainte d'ordre)" (Vandenberghe, 2006, p. 203).

Comme la sociologie critique, la sociologie de la justification met l'accent sur les problèmes de justice sociale, mais sans déshumaniser ses approches au nom d'un structuralisme qui ne voit dans les individus que des pions. Ce qu'elle a de la peine à justifier, c'est l'existence des 'Cités', ce niveau culturel que refusent ceux qui se veulent parfaitement objectifs, comme Bruno Latour.

\section{3- Bruno Latour, théorie de l'acteur-réseau, association et refondation de la sociologie}

On cite toujours Bruno Latour pour les travaux qu'il a consacrés, il y a maintenant plus de trente ans, à la conduite de la recherche dans les laboratoires de sciences naturelles (La Vie de laboratoire, avec Steve Woolgar, 1988/1979). On se réfère moins aux travaux qu'il a consacrés à l'expérience religieuse Uubiler ou les tourments de la parole religieuse, 2002), au droit (La Fabrique du droit. Une ethnographie du Conseil d'Etat, 2002, 2004) ou à la sociologie (Changer de société, refaire de la sociologie, 2005/2006). C'est que le projet épistémologique auquel il s'attache est beaucoup plus vaste qu'on ne le pense généralement, comme l'indique l'Enquête sur les modes d'existence, sous-titrée Une anthropologie des modernes, et qu'il a publié en 2012. Son 
grand projet suscite nombre de commentaires (par exemple : Frédéric Vandenberghe, Complexités du post-humanisme. Trois essais dialectiques sur la sociologie de Bruno Latour, 2006).

A l'origine, Latour s'attache à la sociologie de la science telle que la révèlent les pratiques de laboratoires de sciences naturelles. Son enquête s'élargit progressivement aux sciences sociales. Selon lui, celles-ci ont failli à leur projet : leur façon d'appréhender leur domaine, qui était utile au moment où celui-ci s'est constitué, au XIX ${ }^{\mathrm{e}}$ siècle, s'est figée et ne permet plus de rendre compte du monde qui est en train de naître. Pour classer l'ensemble des observations, on n'avait besoin, il y a un siècle et demi, que de deux catégories, la société et la nature. Elles ne suffisent plus à ordonner ce qui a trait à une réalité plus complexe.

Les sciences sociales telles qu'on les pratique communément mettent en œuvre une double démarche : elles observent ce qui se passe concrètement dans telle ou telle situation, et font appel à un corps d'explicatif développé par ailleurs pour les interpréter et les 'expliquer'. Latour condamne cette démarche duale. Ce qu'il propose est différent :

"Il existe une autre approche, beaucoup moins connue, qui rejette l'axiome fondamental de la première. Dans cette nouvelle façon de voir, on affirme que l'ordre social n’a rien de spécifique ; qu'il n'existe aucune espèce de 'dimension sociale', aucun 'contexte social', aucun domaine distinct de la réalité auquel on pourrait coller l'étiquette 'social' ou 'société' ; qu'aucune 'force sociale' ne s'offre pour 'expliquer' les phénomènes résiduels dont d'autres domaines ne peuvent rendre compte; que les membres de la société savent très bien ce qu'ils font même s'ils ne le verbalisent pas d'une façon qui puisse satisfaire les observateurs ; que les acteurs ne s'inscrivent jamais dans un contexte social et par conséquent, qu'ils sont plus que de 'simples informateurs' ; qu'il est absurde d'ajouter des 'facteurs sociaux' à d'autres disciplines scientifiques" (Latour, 2006, p. 12 ; édition utilisée : La Découverte/poche, 2007).

L'approche que Latour envisage de construire pour succéder à la vieille sociologie porte un nom : c'est la 'sociologie des associations'. Elle repose sur une autre manière de concevoir la société.

"Dans la perspective alternative présentée ici, le 'social' n'est pas une colle capable de tout attacher, y compris ce que d'autres colles ne peuvent pas faire tenir, mais plutôt $c e$ qui est assemblé par de nombreux autres types de connecteurs. Tandis que les sociologues [...] prennent les agrégats sociaux comme un donné susceptible d'éclairer les aspects résiduels de l'économie, de la linguistique, de la psychologie, du management, etc., les chercheurs qui se rattachent à cette seconde perspective considèrent au contraire les agrégats sociaux comme ce qu'il faut expliquer à partir des associations propres à l'économie, à la linguistique, à la psychologie, au droit, au management, etc." (Latour, 2006, p. 12-13).

Comme il le précise, Latour s'adresse aux "enquêteurs désireux de réassembler le social" (Latour, 2006, p. 17). Il leur propose un mode opératoire qu'il a rodé dans ses recherches sur la sociologie des sciences : la théorie de l'acteur-réseau.

"Il faut remplacer le raccourci commode du social par le détour long, ardu et coûteux des associations en changeant également les tâches assignées d'habitude aux sociologues : il n'est plus possible de réduire les acteurs au rôle d'informateurs venant illustrer de façon exemplaire quelque type déjà répertorié ; il faut leur restituer la capacité de produire leurs propres théories sur le social. Notre devoir ne consiste pas à imposer un ordre, à limiter le spectre des entités acceptables, à enseigner aux acteurs ce qu'ils sont, ou à ajouter de la réflexivité à leur pratique aveugle. [...] Il nous faut 'suivre les acteurs eux-mêmes. Ce qui revient à documenter leurs innovations souvent sauvages, afin qu'ils nous apprennent à documenter ce que l'existence collective est devenue entre leurs mains, quelles méthodes ils ont élaborées pour les maintenir, et quels récits sont les plus adaptés pour rendre compte des nouvelles associations qu'ils ont été obligés d'établir" (Latour, 2006, p. 22).

C'est parce que nous vivons dans un monde radicalement différent de celui du XIX ${ }^{\mathrm{e}}$ siècle que les chercheurs doivent recourir à une nouvelle démarche. Celle-ci se singularise d'abord par l'accent qu'elle met sur "les interactions locales, transitoires, face-à-face entre agents dépourvus d'équipement" (Latour, 2006, p. 94). Dès lors :

“[...] A chaque fois que nous voudrons expliquer l'extension dans le temps et dans l'espace d'une interaction quelconque, il va nous falloir en détecter les moyens pratiques. [...] Il existe bien des liens durables, mais cela ne veut pas dire qu'ils sont constitués d'un matériau social - bien au contraire. Nous allons enfin pouvoir découvrir les instruments qui maintiennent les liens en place ; révéler l'ingéniosité constamment requise pour mobiliser des sources nouvelles d'association" (Latour, 2006, p. 95).

Cette recherche conduit à s'attacher aux êtres vivants et aux choses auxquels les gens se lient et par l'intermédiaire desquels ils s'associent les uns aux autres. En voici un exemple :

"Si vous rencontrez un berger et son chien, cela évoquera pour vous des relations sociales. Mais si vous voyez le même troupeau derrière des barbelés, vous vous demandez où sont passés le berger et son chien - pourtant, si les moutons demeurent sa- 
gement dans le pré, c'est que les barbelés remplacent bien les aboiements du chien : qu'un barbelé ait remplacé un chien, voilà bien une relation sociale, et pourtant le chien et le barbelé sont incommensurables aussi bien avant qu'après cette connexion" (Latour, 2006, p. 110-111).

Pour mettre en évidence de telles associations, il convient d'analyser très finement les situations :

"Je vous l'ai dit, notre business à nous, ce sont les descriptions. Tous les autres font du trafic de clichés. Enquêtes, sondages, travail de terrain, archives documentaires, tous les moyens sont bons - on y va, on écoute, on apprend, on pratique, on devient compétent, on modifie nos conceptions. C'est vraiment très simple : cela s'appelle le travail de terrain. Un bon travail de terrain produit toujours de nouvelles descriptions nouvelles" (Latour, 2006, p. 213).

Bruno Latour a fait ses armes en analysant le fonctionnement de laboratoires dans lesquels des expériences étaient menées. Les sciences sociales n'en font pas : les questions et les débats auxquels donnent lieu les observations prennent place à un autre moment, dans un autre contexte, lors de l'écriture :

"Dans notre discipline, le texte n'est pas une histoire, une belle histoire, c'est l'équivalent fonctionnel du laboratoire. C'est là où l'on fait des tests, des expériences et des simulations. Selon ce qui s'y passe, il y a acteur ou non, il y a réseau ou non. Et ça dépend uniquement de la manière précise dont il est écrit - et chaque sujet nouveau exige d'être traité d'une manière nouvelle par un texte spécifique, complètement spécifique “ (Latour, 2006, p. 217).

Ces analyses si minutieuses qu'elles dispensent d'explication peuvent-elles apporter quelque chose aux personnes étudiées ? Non, répond Latour, leur rôle est autre :

"Vous les sociologues du social, vous me sidérez toujours. Si vous étudiez les fourmis [...], est-ce que vous attendriez à ce que votre étude apprenne quoi que ce soit aux fourmis ? Bien sûr que non : elles savent, et vous pas; ce sont elles qui sont les professeurs, et vous l'étudiant. C'est à vous-même que vous expliquez ce qu'elles font, pour votre propre bénéfice ou pour celui d'autres entomologistes, par pour elles qui s'en moquent comme de l'an quarante" (Latour, 2006, p. 219).

Voici donc brièvement résumés les fondements de la démarche que Bruno Latour propose pour construire une nouvelle sociologie, qui ne traite plus du social, mais de ces assemblages qui lient les hommes, les êtres non-humains et les choses ; ceux-ci, qu'il qualifie de collectifs, ne cessent de prendre de nouvelles formes.

"Le social [...] tel qu'on le définit d'habitude n'est qu'un moment particulier dans la longue histoire des assemblages, une forme transitoire dans la quête du corps politique d'une part, et de l'autre, l'exploration du collectif. Le grand projet qui procura son impulsion à la sociologie du social [...] est désormais à bout de souffle. Mais il n'y aucune raison de désespérer. Au contraire, cela veut simplement dire qu'un autre projet, aussi ambitieux que le précédent, doit prendre le relais. Puisque la sociologie du social n'est qu'une façon parmi d'autres de parvenir au collectif, la sociologie des associations reprend la mission de collecter ce que l'idée de social a laissé en suspens" (Latour, 2006, p. 358).

La démarche que propose Bruno Latour a une structure ontologique singulière : elle suppose un monde sans profondeur, sans arrière-plan où pourraient jouer des forces qui échapperaient au commun des mortels :

"En recourant à une métaphore cartographique, on pourrait dire que la sociologie de l'acteur-réseau s'efforce de rendre le monde social aussi plat que possible, afin de s'assurer que l'établissement de tout nouveau lien deviendra clairement visible. " (Latour, 2006, p. 29).

La raison qu'invoque Latour pour ce refus de toute profondeur, c'est de focaliser sur l'essentiel : l'inventaire des liens qui se tissent dans le monde et la multiplicité de leurs configurations et de leurs logiques. Mais son propos va beaucoup plus loin :

"Pour repérer les valeurs multiples et contradictoires auxquelles tiennent ceux qui se disent modernes, il faut accepter qu'il y ait plusieurs régimes de vérité, plusieurs types de raison, plusieurs modes d'existence dont l'enquêteur doit dresser avec soin les conditions de félicité et d'infélicité. On peut alors revisiter le cœur de notre vie collective : les sciences, les techniques, mais aussi le droit, la religion, la politique et, bien sûr, l'économie, la plus étrange et la plus ethnocentrique des productions. Et se poser autrement ces questions : Que nous est-il donc arrivé ? De quoi pouvons-nous hériter ? Qu'avons-nous en propre ? L'enjeu n'est pas mince au moment où les crises écologiques obligent toutes les sociétés à repenser ce qu'elles ont en commun" (Latour, 2012, Quatrième de couverture).

\section{9- De la transdisciplinarité à la métadisciplinarité}

La géographie constitue une des approches possibles pour saisir, comprendre et expliquer le monde, la nature, la société et l'homme ; elle se donne pour objet d'étudier un ensemble de connaissances et de techniques qui permettent de transformer la nature, de modeler l'environnement et de modifier le fonctionnement et le devenir des groupes sociaux. La discipline aborde les mêmes réalités que d'autres approches 
scientifiques, la géologie, la botanique, la zoologie, l'écologie dans le domaine naturel, l'histoire, la sociologie, l'économie, les sciences politiques dans la sphère sociale - mais elle les envisage différemment.

Il y a plusieurs manières de concevoir les rapports entre les disciplines de l'homme et de la société. Certains les mettent toutes sur le même plan et refusent toute préséance entre elles. Dans cette perspective, les sciences entrent en contact par certains points, certaines lignes de contact, certaines aires où elle se superposent, comme l'indiquent ces mixtes que sont, par exemple, l'économie sociale ou la sociologie économique. Il s'agit de secteurs souvent négligés, et qu'il peut être intéressant d'investir. C'est l'idée qui s'était imposée dans les années 1960 et 1970, et qui explique les efforts en faveur de la pluridisciplinarité.

Certaines disciplines de l'homme et de la société seraient plus scientifiques que d'autres : c'est la perspective qu'avait retenue Foucault, qui y voyaient des discours et ne leur conférait pas un statut égal à celui qu'il reconnaissait à la biologie, à l'économie et à la linguistique, parce que ces dernières étaient les seules à traiter du substrat inconscient qui détermine une part essentielle des faits sociaux. Bruno Latour hiérarchise plutôt les disciplines dans le temps : l'économie serait pour lui la discipline première, dans laquelle serait venue s'insérer la sociologie - une étude qui ne serait pas encore parvenue à maturité. Pour Pierre Bourdieu, une hiérarchie existait aussi, dont le plus bas échelon était occupé par la géographie.

L'idée qu'il existe une discipline reine des sciences sociales, qui les coiffent toutes, qui serait plus noble, et qui pourrait au besoin les supplanter, apparaît à divers moments. On a vu qu'elle expliquait l'impérialisme de la sociologie comtienne puis de la sociologie durkheimienne ; certains historiens, comme Marc Bloch, plaçaient également leur discipline à la tête du classement.

Une autre perspective se développe dans les années 1950 et 1960 : celle de l'existence d'une théorie d'ordre supérieur, celle des structures. C'est la capacité à prendre en compte celles-ci qui donnerait à la sociologie critique sa puissance de dévoilement. L'arrogance épistémologique à laquelle conduit une telle interprétation suffit à la condamner.

La conception que nous présentons ici est différente. Elle retient l'idée selon laquelle toutes les disciplines de l'homme et de la société se situent au même niveau, mais elle la justifie différemment : si sciences sociales se situent au même niveau, c'est que chacune correspond à une perspective partielle prise sur un même sujet : l'étude des associations, des collectivités, des communautés, des sociétés dans lesquelles les hommes s'insèrent, et celle des cultures qu'ils ont imaginées pour vivre. C'est parce que ces réalités ne peuvent s'approcher qu'à travers les traces qu'elles laissent qu'elles ont été abordées de manière fragmentaire. Mais toutes les disciplines de la société sont amenées à développer des vues sur ce qu'est l'homme en tant qu'être social, sur la manière dont il est socialisé et dont il se construit, sur les relations qu'il tisse avec ses congénères, sur les institutions qu'il crée et sur l'ordre qu'il réussit à créer et maintenir. Au-delà de la multiplicité des points de vue, il existe donc un corps de connaissances à portée générale que les sciences de l'homme et de la société ont intérêt à partager : c'est un espace méta-disciplinaire ; il importe de prendre conscience de son rôle et de sa fécondité.

La géographie se développe nécessairement dans un climat de concurrence avec d'autres disciplines, ce qui se traduit par d'incessants conflits de frontières ; mais dans le même temps, elle partage avec elles les points de vue et les outils de travail indispensables pour traiter de la nature, de la société, de l'homme : elle comporte des aspects méta-disciplinaires - les idées de milieu, de sélection, de pyramide écologique, d'évolution dans le monde physique, celles de société, d'individu, de décision, de mécanisme de régulation, de pouvoir dans la sphère humaine.

\section{Conclusion}

1- A quoi sert la réflexion épistémologique ? A empêcher les disciplines scientifiques de s'enfermer dans leur tour d'ivoire et à favoriser leur ouverture ? Certainement. Mais son effet a longtemps été aussi de hiérarchiser les sciences : certaines (les mathématiques, l'astronomie, la physique, la biologie dans le domaine des sciences 'dures', l'économie ou la sociologie dans le domaine des sciences sociales) apparaissent alors comme supérieures aux autres et leur servent de modèles. Celles qui ont déjà fait leurs preuves devancent les autres ; ces dernières les suivent avec retard, puisque toutes doivent se développer selon les mêmes lignes.

Transposer ainsi les concepts et les méthodes d'un secteur à l'autre ne va pas sans problème : les sciences de l'homme social et de la société diffèrent des sciences physiques et des sciences naturelles : les distributions et configurations qu'elles appréhendent ne résultent pas directement du jeu de forces générales ; celles-ci s'expriment à travers les décisions d'acteurs qui les prennent en compte, mais en leur accordant un poids qu'ils modulent, ou auquel ils cherchent à échapper. Face à ces difficultés, l'épistémologie des sciences de l'homme social et de la société s'autonomise donc rapidement.

Cette autonomisation se marque de plusieurs façons. 1- Une réflexion émerge sur ce qu'est la société et ce que l'on peut qualifier de social, sur la conscience que l'on prend de la diversité des formes que revêt la vie collective et sur les effets qu'y entraîne la modernisation. 2- La diversité des mécanismes à l'œuvre dans les sociétés est mise en évidence : reproduction à l'identique des cellules closes des mondes premiers, ou inscription dans une histoire qui va dans le sens d'une rationalisation progressive, la rationalité étant 
imposée par des dirigeants et des élites qui guident le mouvement, ou résultant des petites décisions de gens modestes, responsables des lentes dynamiques de la longue durée.

Dans le même temps, les emprunts que les sciences de l'homme social et de la société font aux sciences physiques et naturelles expliquent la vogue que connaissent les interprétations fonctionnalistes ou organicistes. Celles-ci sont progressivement remplacées par des approches plus directement enracinées dans la recherche sociale, et qui mettent à la fois l'accent sur l'existence de structures et le rôle qu'elles jouent, et sur l'influence des représentations et des symboles dans les dynamiques de la vie collective.

Au total, et jusqu'aux années 1960, la réflexion épistémologique témoigne d'un double souci : celui de coller aux spécificités du social, et celui de faire preuve d'une grande rigueur, ce qui conduit les chercheurs à jeter sans cesse des coups d'œil en direction des sciences 'dures' - processus récurrent, dont le dernier épisode se marque par le succès du néo-positivisme logique.

2- Après 1960 ou 1970, l'affaiblissement du rationalisme entraîne des modifications radicales de la réflexion épistémologique. L'évolution était déjà en germe dans l'historisation que l'étude de la Raison connaissait grâce aux travaux de Bachelard, prolongés par ceux de Kuhn. La conviction qu'elle constituait un arrière-plan permanent, un univers des idées à la mode platonicienne, disparaît. Cela explique l'accent nouveau mis sur les pratiques et sur les contextes - dont la théorie de l'acteur-réseau constitue l'exemple le plus emblématique.

L'historisation du rationalisme ouvre la voie à la déconstruction des sciences sociales, qui occupe le devant de la scène de la fin des années 1960 au milieu des années 1980, et qui transpose au domaine de la vie collective un mouvement que Schopenhauer et Nietzsche ont esquissé dès le $\mathrm{XIX}^{\mathrm{e}}$ siècle sur le plan philosophique.

Cette déconstruction souligne le caractère 'situé' des chercheurs en sciences sociales et celui des connaissances qu'ils élaborent. Elle met en avant la part d'inconscient, et donc d'irrationnel, qui caractérise les comportements humains. Elle installe le soupçon dans la pensée sociale. Celle-ci ne doit plus être seulement analytique, c'est-à-dire descriptive et explicative ; il lui faut devenir critique, c'est-à-dire normative et prescriptive.

L'œuvre de déconstruction permet d'en finir avec l'idée que l'évolution des sciences sociales reproduit, mais avec un certain retard, celle des sciences physiques et naturelles. Elle provoque un rapprochement entre sciences sociales et humanités. Elle conduit aussi à l'apparition de disciplines praxéologiques, qui prennent en compte certaines des caractéristiques jusque-là négligées de l'action humaine : (i) l'imparfaite connaissance des situations dans lesquelles les hommes sont amenés à prendre des décisions ; (ii) la volonté qu'ils manifestent de façonner un futur qui préserve leurs intérêts et reflète leurs rêves et leurs valeurs.

3- Mais la déconstruction va plus loin : elle remet en cause les épistémologies de la curiosité et de la raison au motif que ce sont essentiellement des épistémologies du regard - ce qui explique qu'elles débouchent fréquemment sur la surveillance et la domination. Les nouveaux courants font du désir la vraie motivation de beaucoup de connaissances, ce qui conduit à prendre en compte la corporéité des chercheurs et de ceux qu'ils étudient ; c'est désormais à l'ensemble de leur sens que l'on s'attache, et à leur sensualité. Les études queer suivent cette voie.

La déconstruction conduit enfin à la remise en cause de l'idée de société sous les deux variantes qu'elle avait revêtue : le modèle de la communauté et celui de la société. Au-delà, le doute est jeté sur l'idée, dominante depuis le début du XIX ${ }^{\mathrm{e}}$ siècle, selon laquelle les sciences sociales avaient pour objet le lien social - souvent inexistant - et les procédures et institutions qui assuraient l'ordre social.

Cette remise en cause affecte plus directement les disciplines axées sur le lien collectif - la sociologie et l'ethnologie-anthropologie (mais celle-ci se tire d'affaire en se redéfinissant comme une méthodologie des approches microsociales beaucoup plus que comme une science des communautés premières ou traditionnelles). Des disciplines comme la géographie sont également concernées par cette évolution, puisque le glissement des situations de classe vers des situations d'exclusion témoigne d'une transformation dans l'usage que les groupes font de l'espace : on passe d'une cas où il est potentiellement, bien qu'inégalement, ouvert à tous, à un cas où il est exclusivement réservé à certains.

4- La déconstruction n'a qu'un temps et appelle nécessairement une reconstruction, ce à quoi vise une partie de la réflexion épistémologique contemporaine. Dans la sociologie française, que nous avons prise comme exemple, trois voies ont été essayées pour mener à bien cette refondation : la sociologie critique, la sociologie pragmatique de la justification et la théorie de l'acteur-réseau.

Les approches critiques connaissent un immense succès dans toutes les disciplines, mais elles souffrent généralement, comme la sociologie de Bourdieu, de reposer sur une hiérarchie des instances de vérité, qui conduit à attribuer au chercheur des pouvoirs exorbitants.

La sociologie pragmatique de la justification présente beaucoup de parenté avec l'approche culturelle qui se développe en géographie depuis une quarantaine d'années : l'une et l'autre ont la conviction que les individus qu'elles étudient font preuve d'autonomie ; l'une et l'autre étudient les représentations - ou les répertoires de justifications - qu'ils mettent en œuvre. L'histoire, avec l'accent qu'elle place sur les mentalités et les représentations, suit des voies analogues. 
La théorie de l'acteur-réseau présente une structure plus simple que les deux conceptions précédentes, car elle met les hommes, les êtres animés et les choses qu'elle analyse sur le même plan et refuse de voir dans le réel une structure feuilletée. Elle est plus ambitieuse, puisqu'elle vise une reconstruction complète de la réalité appréhendée à la fois dans ses dimensions sociale, naturelle et physique. Elle est plus modeste aussi, dans la mesure où elle observe et constate plus qu'elle ne conjecture.

Prendre en compte l'ensemble des éléments impliqués par la vie collective, hommes, animaux et cadre naturel ou bâti ne peut que convenir au géographe toujours soucieux de la matérialité des situations. La prise en compte des réseaux est pour lui une cause de satisfaction plus grande encore, puisqu'elle conduit à s'attacher, dès le départ, à la dimension spatiale des réalités sociales, qu'ignorait la sociologie classique.

La question se pose évidemment de savoir si la perspective sans profondeur retenue par Bruno Latour sera tenable à la longue. Il conçoit son projet comme première phase d'un processus de reconstruction qui permettra de définir l'objet et les contenus d'un nouveau savoir. La théorie de l'acteur-réseau se présente ainsi comme une utopie scientifique : le savoir qu'elle produit n'est que provisoire et ne prendra tout son sens que lorsqu'on pourra enfin raisonner sur l'ensemble du sujet enfin délimité. L'au-delà que constitue une théorie scientifique en gestation suffira-t-il à motiver longtemps les chercheurs ? N'auraient-ils pas besoin, eux aussi, de prendre en compte le plan des représentations, qui est aussi celui des rêves, de l'imaginaire, de l'utopie comme des religions et des idéologies?

On comprend la difficulté à laquelle se heurte celui qui veut, comme Bruno Latour, respecter les normes d'objectivité de la science : le chercheur a-t-il vraiment accès au plan des représentations ? La réflexion géographique offre peut-être une issue à ce problème. La prise en compte de l'au-delà des images et des rêves transforme l'approche que l'on a du monde, car pour porter un jugement sur le réel observable ici, il faut le comparer à ce qui se passe ailleurs ; c'est grâce à cette démarche que des valeurs sont introduites et que l'action humaine peut revêtir une dimension normative. Les philosophes parlent de transcendance ou d'immanence pour désigner le changement de plan qui fait passer du monde réel à celui des représentations, mais ils ont oublié que ces termes désignent des transformations spatiales. Les géographes sont là pour rappeler que les opérations mentales en question ont toujours existé et qu'elles naissent de l'expérience du paysage. Sous leur forme première, elles naissent en effet de la curiosité que les hommes manifestent pour ce qui se passe au-delà de l'horizon, et de leur aptitude à l'imaginer. Ils créent ainsi des topologies faites d'ailleurs proches ou lointains, passés ou futurs, transcendants ou immanents, conscients ou inconscients. En y logeant leurs représentations, ils donnent un sens à leur existence individuelle, aux destins collectifs qu'ils partagent et au cosmos.

5- La réflexion épistémologique relative aux sciences sociales s'est beaucoup approfondie depuis bientôt un demi-siècle. Elle souligne que les recherches s'y développent à deux niveaux. 1- Elles portent sur les méthodes et les concepts que chaque discipline mobilise selon la perspective qui lui est propre : la géographie pour expliquer la distribution des réalités sociales dans l'espace, l'histoire pour comprendre leur succession dans le temps, la sociologie pour mettre en évidence leur organisation, l'économie pour analyser le circuit des richesses et les sciences politiques pour retracer les formes et le cheminement du pouvoir. Dans cette phase, chaque discipline se distingue nettement des autres. 2- Dans une seconde phase, les chercheurs sont amenés à se préoccuper de la nature et des mécanismes de la société. Ils se retrouvent alors sur un terrain qu'ils partagent : la connaissance de l'homme social et des réalités collectives qu'il construit.

L'évolution contemporaine de l'épistémologie des sciences sociales et de la géographie appelle au développement de ce domaine méta-disciplinaire. Tout laisse présager que sa connaissance s'affermira dans les années qui viennent.

\section{BIBLIOGRAPHIE}

Anderson, Benedict, 1983, Imagined Communities. Reflections on the Origin and Spread of Nationalism, London, Verso.

Bachelard, Gaston, 1934, Le Nouvel Esprit scientifique, Paris, PUF.

Bachelard, Gaston, 1938, La Formation de l'esprit scientifique, Paris, PUF.

Baudelle, Guy, Marie-Vic Ozouf-Marignier et Marie-Claire Robic (dir.), 2001, Géographes en pratiques (1870-1945). Le terrain, le livre, la cité, Rennes, Presses Universitaires de Rennes.

Bhaskar, Roy, 1979, The Possibility of Naturalism, Brighton, Harverster Press, 225 p.

Boltanski, L. et E. Chiapello, 1999/2011, Le Nouvel Esprit du capitalisme, Paris, Gallimard.

Boltanski, Luc et Laurent Thévenot, 1991, De la Justification. Les économies de la grandeur, Paris, Gallimard.

Bourdieu, Pierre, 1982, Ce que parler veut dire : l'économie des échanges linguistiques, Paris, Fayard.

Brunhes J., 1910, La Géographie humaine. Essai de classification positive, Paris, Alcan, 3 vol.

Calbérac, Yann, 2010, Terrains de géographes, géographes de terrain. Communauté et imaginaire disciplinaires au miroir des pratiques de terrain des géographes français $d u \quad X X^{e}$ siècle, Thèse, Université de Lyon-2.

Canguilhem, G., 1977, Idéologie et rationalité, Paris, Vrin. Castoriadis, C., 1975, L'Institution imaginaire de la société, Paris, Seuil.

Chastel, A., 1982, Art et humanisme à Florence au temps de Laurent le Magnifique, Paris, PUF; éd. or., 
Paris, PUF, 1959.

Claval, Paul, 1980, Les Mythes fondateurs des sciences sociales, Paris, PUF.

Claval, Paul, 2010, Les Espaces de la politique, Paris, A.Colin.

Claval, Paul, 2001, Epistémologie de la géographie, Paris, 2001.

Clifford, J., 1988, The Predicament of Culture. Twentieth Century Ethnography, Literature and Art, Cambridge (Mass.), Harvard University Press.

Couteau-Bégarié, H., 1983 Le Phénomène 'Nouvelle Histoire', Paris, Economica.

Cusset, F., 2003, French Theory. Foucault, Derrida, Deleuze \& Cie et les mutations de la vie intellectuelle aux Etats-Unis, Paris, La Découverte.

da Silva, Ana Cristina, 2013, Território e significações imaginárias no pensamento geográfico brasileiro, Goîania, Ediora UFG, $318 \mathrm{p}$.

Deffontaines, Pierre, 1966, . La marque g.ographique des religions ., dans Pierre Deffontaines et Mariel. J. Brunhes-Delamarre (.d.), Géographie générale, Paris, Gallimard, p. 1717-1725.

Delacroix, C., F. Dosse et P. Garcia, 1999, Les Courants historiques en France, Paris, A. Colin.

Deleuze Gilles, Guattari Félis, 1980, Mille Plateaux, Paris, Minuit.

Droysen, J. G., 1855, Historik. Vorlesung über Enzyklopädie und Methodologie der Geschichte. Munich, Oldenburg; 6ème ed., 1971.

Dubet, François, 2006, "Société", in Sylvie Mesure et Patrick Savidan (eds.), Le Dictionnaire des Sciences humaines, Paris, PUF, p. 1094-1097.

Dubet, François et D. Martuccelli, 1998, Dans quelle Société vivons-nous? Paris, Seuil.

Duhem, Pierre, 1913-1917, Système des mondes, Paris.

Dumont, Louis, 1966, Homo hierarchicus, Paris, Gallimard.

Dumont, Louis, 1977, Homo aequalis, Paris, Gallimard.

Durkheim, Emile, 1893, De la Division du travail social, Paris, Alcan.

Durkheim, Emile, 1895, Les Règles de la méthode sociologiques, Paris, Alcan.

Durkheim, Emile, 1912, Les Formes élémentaires de la vie religieuse : le système totémique en Australie, Paris, Félix Alcan.

Ferreti, Federico, 2007, Il Mondo senza la mappa. Elisée Reclus e i geografi anarchici, Milan, Zero in Condotta.

Ferry, Luc, Claude Capelier, 2014, La plus belle Histoire de la Philosophie, Paris, Robert Laffont.

Febvre, Lucien, 1942, Le problème de l'incroyance au XVIe siècle : la religion de Rabelais, Paris, Albin Michel.

Foucault, Michel, 1965, Des mots et des choses, Paris, Gallimard.

Foucault, Michel, 1969, L'Archéologie du savoir, Paris, Gallimard

Foucault, Michel, 1976, Surveiller et punir, Paris, Gallimard.

Giddens, Anthony, 1984, The Constitution of Society, Oxoford, Blackwell, trad. fse, Paris, PUF, 1987.

Hagel, Florence, 2003, "Inclus/exclus", in Jacques Lévy et Michel Lussault (eds), Dictionnaire de la géographie et de l'espace des sociétés, Paris, Belin, p. 493.

Hobbes, Thomas, 1651/1971, Leviathan, London ; trad. fse François Tricaux, Paris, Sirey, 1971

Jullien, François, 2008, De l'Universel, de l'uniforme, du commun et du dialogue entre les cultures, Paris, Fayard.

Kantorowics, Ernst, 1957, The King's two Bodies : a Study in Mediaeval Political Theology, Princeton, Princeton University Press

Koyré, Alexandre, 1966, Etudes d'histoire de la pensée scientifique, Paris, PUF.

Kuhn, Thomas, 1962, The Structure of Scientific Revolutions, Chicago, Chicago University Press.

Latour, Bruno, 2002, Jubiler - ou les tourments de la parole religieuse, Paris, Les Empêcheurs de tourner en rond/le Seuil.

Latour, Bruno, 2004, La Fabrique du droit : une ethnographie du Conseil d'Etat, Paris, La Découverte.

Latour, Bruno, 2006, Changer la Société, refaire la sociologie, Paris, La Découverte ; éd. or. anglaise, Re-assembling the Social. An Introduction to Actor-Network Theory, Oxford, Oxford University Press, 2005.

Latour, Bruno, 2012, Enquête sur les modes d'existence. Une anthropologie des Modernes, Paris, la Découverte.

Latour, Bruno et Serge Woolgar, 1988, La Vie de laboratoire : la production des faits scientifiques, Paris, La Découverte ; éd. or. américaine, 1979.

Lefebvre, Henri, 1968, Le Droit à la ville, Paris, Anthropos. Lefort, Isabelle, 2012, "Le terrain : l'Arlésienne des géographes", Annales de Géographies, vol. 120, n 687-688, sept.-déc., p. 468-486.

Lévi-Strauss, Claude, 1958, Anthropologie structurale, Paris, Plon.1958)

Linton, R., 1968, De l'homme, Paris, Editions de Minuit; éd. or. américaine, 1936.

Lyotard, François, 1979, La Condition postmoderne. Rapport sur le savoir, Paris, Editions de Minuit.

Manilowski, Bronislaw, 1963/19212, Les Argonautes du Pacifiques occidental, Paris, Gallimard.

Martuccelli, D., 1999, Sociologie de la modernité, Paris, Gallimard.

Merleau-Ponty, Maurice, 1945, Phénoménologie de la Perception, Paris, Gallimard.

Meyerson, Emile, 1921, De l'Explication dans les sciences, Paris.

Montbrial, Thierry de, 2003, L'Action et le système du monde, Paris PUF.

Montbrial, Thierry de, 2006, Géographie politique, Paris, PUF.

Nisbet, Robert, 1966, The Sociological Tradition, New York, Basic Books.

Parsons, Talcott, 1951, The Social System, Glencoe, The Free Press.

Paugam, Serge (dir.), 1996, L'Exclusion. L'état des savoirs, Paris, La Découverte.

Ratzel Friedrich., 1882-1891, Anthropogeographie, oder Anwendung der Erdkunde auf die Geschichte, Stuttgart, Engleborn, 2 vol.

Ricœur, Paul, 1985, . Mythe. C- L'interpr.tation philosophique ., dans Encyclopædia Universalis, t. 12, p. 883-890.

Robertson, Iain and Penny Richards (eds.), 2003, Studying Cultural Landscapes, London, Arnold.

Robic, Marie-Claire (dir.), 2000, Le "Tableau de la géographie de la France" de Paul Vidal de la Blache, Paris, Editions du CTHS. 
Sibley, David, 1995, Geographies of exclusion, Londres, Routledge.

Siegfried, André, 1913, Tableau de géographie politique de la France de l'Ouest, Paris, A. Colin

Tönnies, Ferdinand, 1887, Gemeinschaft und Gesellschaft, trad. fse, Communauté et société, Paris, PUF, 1944, Retz-CEPL, 1977.

Vandenberghe, Frédéric, 1997-1998, Une Histoire critique de la sociologie allemande, Paris, La Découverte, 2 vol. Vandenberghe, Frédéric, 2006, Complexité du posthumanisme. Trois essais dialectiques sur la sociologie de Bruno Latour, Paris, L'Harmattan.

Varii Auctores, 1996, "L'exclusion. Constructions, usages, épreuves"(numéro thématique) , Politix $n^{\circ} 34$, second trimestre.

Volvey, A., Calbérac, Y., Houssay-Holschuch, M., 2012, "Introduction générale", in : Terrains de Je, Annales de Géographies, vol. 120, n 687-688, sept.-déc., p. 441-461.

Zelinsky, Wilbur, 2001, "The World and its identity crisis", p. 129-149 de : Adams, Paul et al. (eds), Textures of Place. Exploring Humanist Geographies, Minneapolis, University of Minnesota Press. 